\title{
Effect of pinning on the vortex-lattice melting line in type-II superconductors
}

\author{
G. P. Mikitik ${ }^{1,2}$ and E. H. Brandt ${ }^{1}$ \\ ${ }^{1}$ Max-Planck-Institut für Metallforschung, D-70506 Stuttgart, Germany \\ ${ }^{2}$ B. Verkin Institute for Low Temperature Physics 83 Engineering, \\ Ukrainian Academy of Sciences, Kharkov 61103, Ukraine
}

(Dated: October 14, 2018)

\begin{abstract}
The vortex-lattice melting line in three-dimensional type-II superconductors with pinning is derived by equating the free energies of the vortex system in the solid and liquid phases. We account for the elastic and pinning energies and the entropy change that originates from the disappearance of the phonon shear modes in the liquid. The pinning is assumed to be caused by point defects and to be not too strong so that the melting line lies inside the so-called bundle-pinning region. We show that the derived equation for the melting line is equivalent to some Lindemann criterion, which however differs from that used previously. Estimating the effect of pinning on the entropy jump at melting, we find the upper critical point of the melting line from the condition that this jump vanishes. We also consider the $H-T$ phase diagrams of type-II superconductors for different strengths and types of pinning and analyze the two recently discussed scenarios how the melting line and the order-disorder line merge.

PACS numbers: 74.25.Qt, 74.72.Bk
\end{abstract}

\section{INTRODUCTION}

In three-dimensional high- $T_{c}$ superconductors with pinning, two phase transition lines are known to exist in the magnetic field $H$ - temperature $T$ plane: $\frac{1,2,3.4 .5}{1.7}$ The line $H_{m}(T)$ where a quasiordered Bragg glass 6.7 thermally melts into a flux-line liquid, and the order-disorder transition line $H_{\text {dis }}(T)$ separating the Bragg glass from an amorphous vortex state. The melting is caused by thermal vibrations of the lattice, while the order-disorder transition is induced by quenched disorder in the vortex system. These two lines merge at some point in the $H-T$ plane. Although both transitions are accompanied by a proliferation of dislocations in the vortex lattice, it was $\operatorname{argued}^{8}$ that the dislocation density $\rho$ is essentially different in these cases: $\rho \sim a^{-2}$ for melting, and $\rho \sim R_{a}^{-2}$ for the order-disorder transition. Here $a$ is the spacing between flux lines, and $R_{a}$ is the so-called positional correlation length ${ }^{9}$ within which the relative vortex displacements caused by the quenched disorder are of the order of $a$. In fact, an intersection of these two different phase transition lines occurs in this scenario, and the order-disorder line terminates at the intersection point while the melting line continues for some distance to higher $H$, see Fig. 10 Within this physical picture, the existence of the so-called slush phase $\frac{10}{13}$ can be naturally explained. Recent experiments11.12.13.14.15 for $\mathrm{YBaCuO}$ seem to support this scenario. On the other hand, experimental data 16.17 .18 for BSCCO strongly argue in favor of a different scenario which was implied, e.g., in the Refs. 25 . In this second scenario, the dislocation densities for both lines coincide at the point where these lines merge, and in fact, one deals with only one phase transition line that describes both the order-disorder transition at low temperature and the melting near $T_{c}$, Fig. 1

Phase diagrams of superconductors with pinning reflect the competition of three characteristic energies ${ }^{3}$ : the elastic energy, the pinning energy, and the energy of thermal fluctuations. At melting, the cost in the elastic energy due to the proliferation of dislocations is mainly balanced by the entropy gain associated with thermal fluctuations, while the role of the pinning energy, as shown below, is determined by the parameter $a / R_{c}$. Here $R_{c}$ is the transverse collective pinning length ${ }^{9}$. On the other hand, at the order-disorder transition, the balance of pinning energy and elastic energy is most important, while the relative contribution of the entropy gain is negligible at low temperatures and, according to the scenario of Ref. 8 , is determined by the ratio $a / R_{a}$ near the intersection point. Thus, if the intersection of the melting and the order-disorder lines occurred sufficiently deep in the bundle pinning region (so that $R_{c} \gg a$ at this point), the scenario of Ref. 8 would lead to the conclusion that up to the intersection point, one can find the melting line by neglecting the pinning, and the order-disorder line by neglecting the entropy gain. Just this approximation was used in our paper ${ }^{4}$ for analyzing the phase diagrams of superconductors. However, our recent results 19 point out that flux-line pinning can affect the melting line near the intersection point since at this point, the ratio $R_{c} / a$ has decreased to several units for any magnitude of the quenched disorder in the vortex lattice (even when the disorder is weak). As to the second scenario, the three energies are all the same order of magnitude in the temperature region where the order-disorder transition gradually transforms into melting. Thus, whatever scenario occurs in reality, a detailed investigation of the effect of pinning on the melting line is important to clarify the most intriguing part of the phase diagram.

The effect of pinning by point defects on the melting line was observed both in $\mathrm{BSCCO}^{20}$ and in $\mathrm{YBaCuO}^{21,22,23}$ crystals. It was discovered that an increase of the quenched disorder in the vortex lattice leads to a noticeable shift of the intersection point to lower 
magnetic fields and simultaneously pushes the melting line in the $T-H$ plane slightly downwards, i.e., at a fixed temperature the appropriate magnetic field of the melting, $H_{m}(T)$, decreases. It is important that the shift of the intersection point is essentially more pronounced than the decrease of $H_{m}(t)$ itself.

Some theoretical results on this subject were obtained in Refs. 25 24. Larkin and Vinokur ${ }^{24}$ started from the assumption that for the vortex lattice with quenched disorder to melt, the temperature must match a characteristic barrier composed of the elastic energy, $E_{\mathrm{el}}$, and the pinning energy, $E_{\text {pin }}$. So they estimated the effect of pinning on the melting line by considering the following balance of these three energies:

$$
T=E_{\mathrm{pin}}+E_{\mathrm{el}},
$$

where $E_{\text {pin }}$ and $E_{\text {el }}$ were calculated in the so-called cage model $\underline{1}$ But it follows from this equation that the melting line has to shift upwards when the quenched disorder increases. Another approach was used in Ref. 2. To describe the unified phase transition line, Giamarchi and Le Doussal, ${ }^{2}$ who implied the second scenario, put forward the following generalization of the Lindemann criterion:

$$
u_{\text {total }}^{2}=c_{L}^{2} a^{2},
$$

where $c_{L}$ is the Lindemann constant $\left(c_{L} \sim 0.1-0.2\right)$, and $u_{\text {total }} \sim\left[2 u_{T}^{2}+u^{2}(a, 0)\right]^{1 / 2}$ is the rms displacement of neighboring flux lines caused both by the thermal fluctuations and by the quenched disorder in the lattice. Here $u_{T}$ is the magnitude of the thermal fluctuations, while $u(a, 0)$ describes the mean relative displacement of neighboring flux lines caused by the disorder. This criterion leads to the usual condition for the order-disorder transition ${ }^{1,3.4}$ at low temperatures when $u_{T}$ is negligible, and it goes over to the well known Lindemann criterion for pure melting when the disorder disappears. Equation (2) results in a qualitatively correct dependence of the melting line on pinning by point defects. However, this reasonable criterion is only an interpolation formula between the two limiting cases and has no serious justification. The same is true for the criterion of Ref. 5 . Radzyner et al,$^{\frac{5}{}}$ described the unified phase transition line using the criterion,

$$
u_{T}^{2}+u^{2}(a, 0)=c_{L}^{2} a^{2},
$$

which practically coincides with Eq. (2). The criterion (3i) is equivalent to the following balance of energies:

$$
T+E_{\text {pin }}=E_{\mathrm{el}},
$$

which evidently differs from Eq. (11). In calculations of the phase transition line, the energies $E_{\text {pin }}$ and $E_{\text {el }}$ were estimated in Ref. 5 in the framework of the cage model. But it remained unclear why the pinning energy $E_{\text {pin }}$ now enters into Eq. (4) with the opposite sign as compared to the energy balance (2) suggested by Larkin and Vinokur $\stackrel{24}{\underline{4}}$

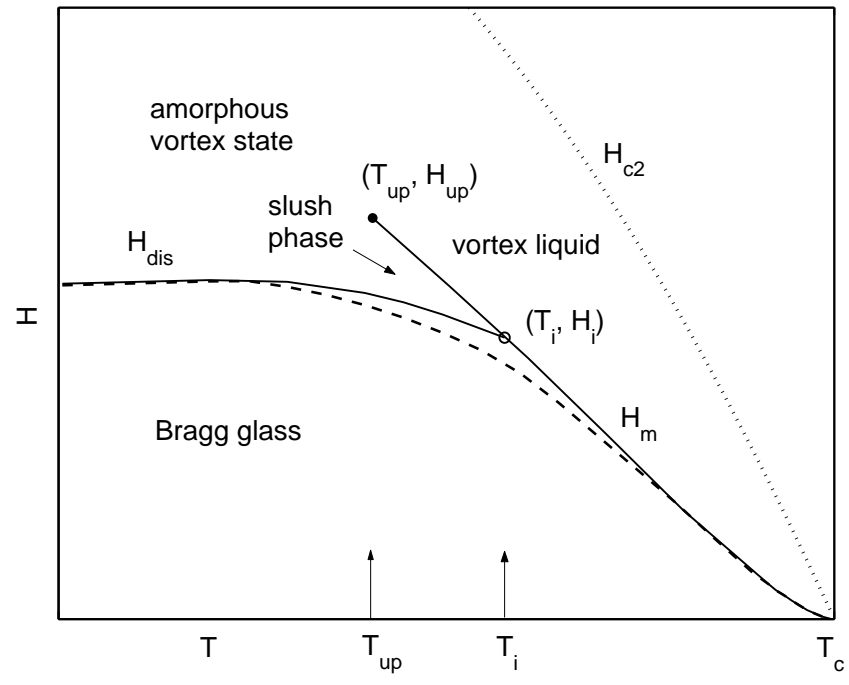

FIG. 1: Schematic $T-H$ phase diagrams for the first (solid lines) and for the second (dashed line) scenarios. In the first scenario the melting line terminates at the so-called upper critical point $\left(T_{\mathrm{up}}, H_{\mathrm{up}}\right)$ which in general does not coincide with the intersection point $\left(T_{i}, H_{i}\right)$. In this case a slush phase (i.e., a vortex liquid with smaller density of dislocations) can be observed. In the second scenario the order-disorder and the melting lines are manifestations of a unified phase transition line. Note that for both scenarios the vortex liquid and the amorphous vortex state are, in fact, one and the same phase, which has different viscosity at low and high temperatures. (We do not discuss the "vortex glass transition" which may be not a true phase transition.)

In the present paper, in order to find and to justify a criterion for the melting of the flux-line lattice with quenched disorder, we start with the analysis of melting in the ideal lattice and show that three different approaches lead to the same dependence $H_{m}(T)$. These approaches are: the Lindemann criterion, the energy balance, and the rigorous approach based on the GinzburgLandau (or on the London) Hamiltonian. In the case of the lattice with quenched disorder, we show that the pinning energy in the flux-line liquid is larger than the pinning energy in the Bragg glass. For this reason, the difference of these pinning energies, $E_{\text {pin }}$, has the opposite sign as compared to Eq. (11), and we arrive at an equation similar to Eq. (4) but with an expression for $E_{\text {pin }}$ that differs from the estimates previously published. Besides this, we estimate the effect of pinning on the entropy gain at melting and find the upper critical point of the melting line from the condition that this gain vanishes. We also show that the result for $H_{m}(T)$ based on this energy balance agrees with the result which can be derived from the Ginzburg-Landau Hamiltonian. Then, using the $H_{m}(T)$ obtained in the framework of the second and the third approaches, we find how the Lindemann criterion should be modified to give the same melting line. Interestingly, the presented energy balance clarifies the difference between the first and the second scenarios. Finally, we present 
the $T$ - $H$ phase diagrams of superconductors with two types of flux-line pinning by point defects and compare these diagrams for the two scenarios.

In this paper we consider only magnetic fields exceeding considerably the lower critical field $H_{c 1}$ and thus do not distinguish between the magnetic field $H$ and the magnetic induction $B$. Besides this, we deal only with uniaxial anisotropic three-dimensional superconductors, neglecting completely the decoupling of the superconducting layers. The anisotropy is characterized by the parameter $\epsilon=\lambda_{a b} / \lambda_{c}<1$ where $\lambda_{a b}$ and $\lambda_{c}$ are the London penetration depth in the plane $a b$ perpendicular to the anisotropy axis and along this axis, respectively. The magnetic field is assumed to be directed along the anisotropy axis. As to the quenched disorder in the fluxline lattice, we assume that it is caused by point defects and is not too strong such that the melting line lies entirely in the bundle pinning region.

\section{MELTING OF THE IDEAL VORTEX LATTICE}

We begin with the analysis of melting in the ideal pinning-free vortex lattice and compare the results of various approaches.

\section{A. Lindemann criterion}

According to the well known Lindemann criterion, the flux-line lattice melts when the magnitude of the thermal displacements of the lattice relative to its equilibrium position, $u_{T}$, reaches a certain fraction of the spacing between the flux lines, $a$ :

$$
u_{T}^{2}=c_{L}^{2} a^{2}
$$

where $a=\left(\Phi_{0} / H\right)^{1 / 2}, \Phi_{0}$ is the flux quantum, and $c_{L}$ is the Lindemann constant. The magnitude $u_{T}$ depends on the elastic moduli of the lattice ${ }^{25}$ and was calculated in many papers; see, e.g., Refs. 9, 25 26 27 28. It can be represented in the form:

$$
u_{T}^{2} \approx \xi^{2} \cdot t\left(\frac{G i}{1-t^{2}}\right)^{1 / 2} h^{-1 / 2} f(h),
$$

where $\xi(t)$ is the coherence length in the $a b$ plane, $h=$ $H / H_{c 2}(t), t=T / T_{c}, H_{c 2}(t)=\Phi_{0} / 2 \pi \xi^{2}$ is the upper critical field, $G i$ is the Ginzburg number,

$$
G i=\frac{1}{2}\left(\frac{T_{c}}{H_{c}^{2} \epsilon \xi_{0}^{3}}\right)^{2}
$$

which characterizes the width of the fluctuation region in zero magnetic field, $\xi_{0}$ and $H_{c}$ are the coherence length and thermodynamic critical magnetic field of the superconductor in the Ginzburg - Landau theory extrapolated to $T=0$. For definiteness, we implied in Eq. (6) and below that $\xi^{2}(t)=\xi^{2}(0) /\left(1-t^{2}\right)$. [Hence $\xi_{0}=\xi(0) / \sqrt{2}$ ]. The complete expression for the function $f(h)$ was given in Ref. 28, but for our further analysis it is sufficient to use a simplified form of this function ${ }^{4}$ in which the contribution containing the compression modulus of the vortex lattice, $c_{11}$, is neglected:

$$
f(h)=\frac{2 \beta_{A}}{1-h} \frac{\left[1+(1+\tilde{c})^{2}\right]^{1 / 2}-1}{\tilde{c}(1+\tilde{c})},
$$

with $\tilde{c}=0.5\left[\beta_{A}(1-h)\right]^{1 / 2}$, and $\beta_{A}=1.16$. Note that this formula can be rewritten in the form:

$$
f(h)=\frac{f_{1}(h)}{(1-h)^{3 / 2}},
$$

where the function $f_{1}(h)$ defined by this equality decreases monotonically with increasing $h$, and its variation in the interval $0<h<1$ is not large: $f_{1}(0) \approx 2.34$, $f_{1}(1) \approx 1.78$. Thus, to a first approximation, this function can be considered as a constant, $f_{1}(h) \approx f_{1} \sim 2$.

Combining formulas (5)-(8), we arrive at the equation for the normalized melting field $h_{m}(t)=H_{m}(t) / H_{c 2}(t)$ :

$$
t\left(\frac{G i}{1-t^{2}}\right)^{1 / 2} h_{m}^{1 / 2} \frac{f_{1}\left(h_{m}\right)}{\left(1-h_{m}\right)^{3 / 2}}=2 \pi c_{L}^{2} .
$$

This equation agrees with those obtained earlier 9.25.26.27.28 and differs from them only in the form of the function $f_{1}(h)$ since different authors used slightly different approximations for the elastic moduli or took into account the contribution associated with the compression modulus of the vortex lattice.

When the normalized melting field $h_{m}(t)$ is small, $h_{m} \ll 1$, it follows from Eq. (9) that

$$
\frac{H_{m}(t)}{H_{c 2}(0)}=\left(\frac{2 \pi c_{L}^{2}}{f_{1}(0) t}\right)^{2} \frac{\left(1-t^{2}\right)^{2}}{G i}
$$

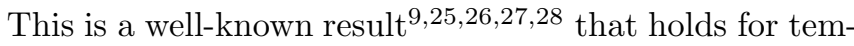
peratures near $T_{c}$ (but outside the fluctuation region in zero magnetic field), namely for

$$
G i \ll 1-t^{2} \ll\left(f_{1}(0) / 2 \pi c_{L}^{2}\right)^{2} G i .
$$

Note that for inequalities (11) to be fulfilled in a sufficiently wide temperature interval, the Ginzburg number should not be too small. In the opposite limiting case,

$$
1-t^{2} \gg\left(f_{1}(1) / 2 \pi c_{L}^{2}\right)^{2} G i
$$

the field $h_{m}$ is close to unity, and one obtains from Eq. (9):

$$
1-h_{m} \approx\left(\frac{f_{1}(1)}{2 \pi c_{L}^{2}}\right)^{2 / 3} t^{2 / 3}\left(\frac{G i}{1-t^{2}}\right)^{1 / 3}
$$

or equivalently,

$$
\frac{H_{c 2}(t)-H_{m}(t)}{H_{c 2}(0)} \approx\left(\frac{f_{1}(1)}{2 \pi c_{L}^{2}}\right)^{2 / 3} t^{2 / 3} G i^{1 / 3}\left(1-t^{2}\right)^{2 / 3} .
$$


Note that $H_{c 2}(0) G i^{1 / 3}\left(1-t^{2}\right)^{2 / 3}$ is the width (along the $H$ axis) of the fluctuation region in not too small magnetic fields, ${ }^{29,30,31} H \gg G i H_{c 2}(0)$, and for inequality (12) to hold, the Ginzburg number should not be too large.

\section{B. Energy balance}

At melting, proliferation of dislocations occurs in the vortex lattice. These dislocations create a network in the lattice, and we consider a mean unit cell of this network composed of edge and screw dislocations. The energies of these dislocations are of the order of

$$
\begin{aligned}
E_{\text {edge }} & \sim c_{66} a^{2} l_{\|}, \\
E_{\text {screw }} & \sim\left(c_{44} c_{66}\right)^{1 / 2} a^{2} l_{\perp},
\end{aligned}
$$

where $c_{66}$ and $c_{44}$ are the shear and tilt moduli of the flux-line lattice, while $l_{\|}$and $l_{\perp}$ are the dimensions of the cell in the longitudinal and transverse directions to $H$, respectively. Since at the melting these dimensions are of the order of $a$ (see below), we have omitted the logarithmic factors $\ln \left(l_{\|} / a\right), \ln \left(l_{\perp} / a\right)$ in the above formulas for the dislocation energies. The shear and tilt moduli may be expressed as, 9.25

$$
\begin{aligned}
c_{66} & \approx \frac{\varepsilon_{0}}{4 a^{2}}(1-h)^{2}, \\
c_{44} & \sim \frac{\epsilon^{2} \varepsilon_{0}}{a^{2}}(1-h),
\end{aligned}
$$

where $h=H / H_{c 2}(t), \varepsilon_{0}=\left(\Phi_{0} / 4 \pi \lambda_{a b}\right)^{2}$, and we have omitted the logarithmic factor of type $\ln (a / \xi)$ in the tilt modulus (this nonlocal modulus should be estimated at wave vectors $k$ of the order of $\left.l_{\|}^{-1}, l_{\perp}^{-1}\right)$. The factors containing $(1-h)$ take into account the softening of the vortex lattice near the $H_{c 2}(t)$ line ${ }^{32}$ Minimization of the elastic energy of the cell at its fixed volume leads to $E_{\text {edge }} \sim E_{\text {screw }}$ and hence gives the relation between $l_{\|}$and $l_{\perp}$ :

$$
l_{\|} \sim l_{\perp}\left(\frac{c_{44}}{c_{66}}\right)^{1 / 2} .
$$

Thus, up to a numerical factor, the elastic energy of the dislocation cell, $E_{\text {el }}$, equals $E_{\text {screw }}$.

At melting, the cost in the elastic energy due to the proliferation of the dislocation network is balanced by the entropy gain in the free energy of the flux-line lattice. We now give a simple estimate of this gain: The vortexlattice degrees of freedom associated with shear undergo a change when the melting occurs. This change occurs for lattice modes with wave-lengths greater than $l_{\|}, l_{\perp}$. There are $l_{\|}^{-1} l_{\perp}^{-2}$ modes of this type in the unit volume of the lattice, and each of them contributes about $T$ to the entropy gain. Thus, up to a numerical factor, the gain per cell of the dislocation network is $T \cdot l_{\|}^{-1} l_{\perp}^{-2} \cdot l_{\|} l_{\perp}^{2} \sim T$.
We now can write down the change of the free energy per cell at melting:

$$
\Delta F \propto C\left(c_{44} c_{66}\right)^{1 / 2} a^{2} l_{\perp}-T,
$$

where some constant $C$ is the ratio of the unknown numerical factors in $E_{\mathrm{el}}$ and in the entropy gain. Minimization of Eq. (18) with respect to the parameter $l_{\perp}$ leads to the conclusion that this parameter should have the minimum possible value. It is clear that this value is of the order of $a$ in the lattice. Then, taking into account that $\Delta F=0$ at the melting, we arrive at

$$
C\left(c_{44} c_{66}\right)^{1 / 2} a^{3}-T=0,
$$

where this constant $C$ may be slightly renormalized as compared to the $C$ of Eq. (18). Inserting Eqs. (16) for the elastic moduli and assuming $\lambda_{a b}(t) / \xi(t)=$ const, one finds the equation for the melting line $h_{m}(t)$ :

$$
t\left(\frac{G i}{1-t^{2}}\right)^{1 / 2} \frac{h_{m}^{1 / 2}}{\left(1-h_{m}\right)^{3 / 2}}=\frac{\sqrt{\pi} C}{4} .
$$

As was noted earlier (see, e.g., Refs. 3, 9), formula (10) for the melting line near $T_{c}$ can be obtained from the energy balance. Here we have taken into proper account the softening of the elastic moduli near $H_{c 2}(T)$, and now equation (20) shows that not only formula (10) but also expression (13) can be derived by this method. Moreover, if one uses the approximation in which the function $f_{1}(h)$ is a constant, ${ }^{33} f_{1}(h) \approx f_{1}$, and put $C=8 \sqrt{\pi} c_{L}^{2} / f_{1}$, Eqs. (9) and (20) completely coincide in the whole temperature interval.

\section{Some exact results}

Within the mean-field theory, when one neglects fluctuations of the superconducting order parameter, the melting line $H_{m}(T)$ coincides with the $H_{c 2}(t)$ line. It is the fluctuations that shift $H_{m}(t)$ downwards in the $H$-T plane. As was mentioned above, see Eq. (14), the Lindemann criterion shows that at sufficiently strong magnetic fields, the distance between the melting line and the mean-field $H_{c 2}(t)$ line is comparable with the width of the fluctuation region. But then the question arises about the applicability of this criterion (and of the energy balance) for determining $H_{m}$ in this region of the magnetic fields since expressions (16) for the elastic moduli were derived in the framework of the mean-field theory without accounting for the fluctuations. In this context, it should be noted that one cannot confine oneself to taking into account only the first fluctuation correction to the elastic moduli (in the fluctuation amplitude) since inside the fluctuation region the amplitude is large, and corrections of all orders are essential. In particular, the renormalized elastic moduli will vanish on a line which differs from the mean-field $H_{c 2}(t)$. However, simple considerations ${ }^{30.34}$ show that at $1-h_{m} \ll 1$, strong fluctuations can only 
renormalize the numerical factor in Eq. (13), but the dependences of $H_{m}$ on $t$ and on $G i$ remain unchanged. We now briefly outline these considerations.

In dimensionless units the Ginzburg-Landau Hamiltonian depends on the three parameters: $t, H / H_{c 2}(0)$ and the Ginzburg number Gi. As well known, the quadratic part (in the order parameter) of this Hamiltonian looks like the Hamiltonian of a particle with double electron charge in a magnetic field, and the energy spectrum of this particle is the so-called Landau levels. It is essential that in fields $H \gg G i H_{c 2}(0)$, the distance between these levels exceeds the width of the fluctuation region. Thus, if one expands the order parameter into the eigenfunctions of the particle, only the modes of the order parameter corresponding to the lowest Landau level strongly fluctuate near the melting line, and one may retain only these modes in the Hamiltonian to calculate the fluctuation part of the free energy of a superconductor. It turns out that the Hamiltonian thus obtained depends on a single combination of the parameters. In our notations, this combination, $Q$, can be represented in the form:

$$
Q=\frac{(1-h)\left(1-t^{2}\right)^{1 / 3}}{t^{2 / 3} G i^{1 / 3} h^{2 / 3}},
$$

where $h=H / H_{c 2}(t)$. Hence, in this region of the magnetic fields, the free energies of the vortex liquid, $F_{\text {liq }}$, and the vortex lattice, $F_{\text {lat }}$, are also determined only by this combination, and for the melting line $h_{m}(t)$ we arrive at the equation:

$$
F_{\text {liq }}(Q)=F_{\text {lat }}(Q) .
$$

The solution of this equation has the form:

$$
Q=\frac{\left(1-h_{m}\right)\left(1-t^{2}\right)^{1 / 3}}{t^{2 / 3} G i^{1 / 3} h_{m}^{2 / 3}}=C_{1},
$$

where $C_{1}$ is some constant. Taking into account that $h_{m} \approx 1$, we find from equation (23):

$$
1-h_{m} \approx C_{1} t^{2 / 3}\left(\frac{G i}{1-t^{2}}\right)^{1 / 3} .
$$

It is seen that up to a numerical factor, this expression indeed coincides with Eq. (13).

The above consideration does not use any perturbation theory and therefore is exact, but it does not yield the constant $C_{1}$. Using some variants of perturbation theory (see, e.g., Ref. 35), approximate expressions for $F_{\text {liq }}$ and $F_{\text {lat }}$ were obtained in Refs. 36, 37. On this basis, an equation for the melting line, which agrees with Eq. (23), was derived in these papers together with the appropriate constant $C_{1}$. Hikami et al ${ }^{36}$ estimated $C_{1} \approx 7$, while $\mathrm{Li}$ and Rosenstein ${ }^{37}$ who used a refined expression for $F_{\text {lat }}$ found $C_{1} \approx 9.5$.

A consideration similar to that presented above was also applied to the Hamiltonian of the vortex system in the London approximation, see Ref. 38 . In the region of the magnetic fields considered here, $H \gg H_{c 1}$, one has $\lambda_{a b} \gg a$, and the Hamiltonian, as well as the free energy of the vortex system, are determined by a single combination of the physical parameters: $\epsilon \varepsilon_{0} a / T$. Thus, the melting line $H_{m}(T)$ is found from the equation:

$$
\frac{\epsilon \varepsilon_{0}(T) a\left(H_{m}\right)}{T}=C_{2}
$$

with some constant $C_{2}$. In other words, the London approximation leads to $H_{m}(t) \propto\left[t \lambda_{a b}^{2}(t)\right]^{-2}$. Putting $\lambda_{a b}(t)=\lambda_{a b}(0)\left(1-t^{2}\right)^{-1 / 2}$ and taking into account the definition of $G i$, we arrive at the formula:

$$
\frac{H_{m}(t)}{H_{c 2}(0)}=\frac{\pi}{\left(2 C_{2} t\right)^{2}} \frac{\left(1-t^{2}\right)^{2}}{G i}
$$

which agrees with Eq. (10).

To summarize, we have shown in this section that up to numerical factors, the three different approaches lead to the same dependences of the vortex-lattice melting field $H_{m}$ on the temperature $T$ and on the Ginzburg number Gi, Eqs. (10) and (14). Thus, after obtaining these dependences, e.g., from the energy balance, for the vortex lattice with pinning, one can guess the true form of the Lindemann criterion for this lattice. We shall use this procedure in the next section.

\section{MELTING OF THE VORTEX LATTICE WITH QUENCHED DISORDER}

In this section we analyze the melting line of the vortex lattice with pinning assuming that the line is in the bundle pinning region (where the transverse collective pinning length $R_{c}$ is greater than $a$ ). As it follows from the figures of Sec. IV B, the melting line, as a rule, does entirely lie in this region, and only in the case of sufficiently strong $\delta T_{c}$ pinning can it enter the single vortex pinning region.

\section{A. Energy balance}

We now consider the influence of pinning by point defects on the energy balance. The adjustment of the vortex system to the pinning potential decreases the total energy of the system, and the amount of this decrease is just of the order of the pinning energy. It will be shown in this section that at melting, the pinning energy in the flux-line liquid, $E_{\text {pin }}^{\text {liq }}$, is noticeably greater than the pinning energy in the flux-line lattice (Bragg glass), $E_{\text {pin }}^{\text {lat }}$. Thus, there is a gain in the free energy of the vortex system, $E_{\text {pin }}$, associated with the pinning energy: $E_{\text {pin }}=E_{\text {pin }}^{\text {liq }}-E_{\text {pin }}^{\text {lat }} \sim E_{\text {pin }}^{\text {liq }}$. This gain adds to the entropy gain, and equation (18) is modified as follows:

$$
\Delta F \propto C\left(c_{44} c_{66}\right)^{1 / 2} a^{2} l_{\perp}-T \Delta S-E_{\mathrm{pin}},
$$


where the factor $\Delta S$ takes into account the effect of pinning on the entropy gain per dislocation cell.

In order to estimate $E_{\text {pin }}^{\text {lat }}, E_{\text {pin }}^{\text {liq }}$ and $\Delta S$, it is necessary in general to take into account the so-called thermal depinning 9.39 since the thermal displacement $u_{T}$ is sufficiently large at the melting, $u_{T} \sim c_{L} a$. However, to explain the main ideas, we first carry out the estimates neglecting this depinning and then generalize the obtained results by taking it into account.

The pinning energy of the flux-line lattice without dislocations, i.e., of the Bragg glass, in the volume equal to the cell of the dislocation network, $E_{\text {pin }}^{\text {lat }}$, can be estimated using the results of collective pinning theory ${ }^{9}$

$$
E_{\mathrm{pin}}^{\mathrm{lat}} \sim\left(W l_{\|} l_{\perp}^{2}\right)^{1 / 2} u
$$

where $W=f_{\text {pin }}^{2} n \xi^{2} / a^{2}, f_{\text {pin }}$ is the mean elementary pinning force exerted by one point defect, $n$ is the concentration of the defects, and the expression $\left(W l_{\|} l_{\perp}^{2}\right)^{1 / 2}$ is the mean pinning force per cell. The displacement $u \equiv u\left(l_{\perp}, 0\right) \sim u\left(0, l_{\|}\right)$is the rms relative shift of two line elements in the vortex lattice separated by a distance $l_{\perp}$ transverse to the magnetic field, or by $l_{\|}$along the field. This shift is caused by the random point defects. The magnitude of $u$ can be expressed in terms of the transverse collective pinning length, $R_{c}$, at which the relative displacements of points in the lattice are of the order of $\xi$. In particular, if the small bundle pinning regime occurs, i.e., $R_{c}<\lambda_{a b}$, one has: $\frac{9}{9}$

$$
u^{2} \approx \frac{\xi^{2}}{1+\ln \left(R_{c}^{2} / a^{2}\right)},
$$

where we have used that $l_{\perp} \sim a$. Note that according to Eq. (17), the longitudinal dimension $l_{\|}$is of the order of $\epsilon a /(1-h)^{1 / 2}$.

At melting, proliferation of dislocations occurs, and in the liquid vortices can adjust themselves to the pinning potential not only via their elastic deformations as in the Bragg glass but also via the plastic vortex-lattice displacements generated by dislocations. This additional adjustment mechanism increases the pinning energy in the liquid. To estimate the pinning energy per dislocation cell in the liquid, $E_{\mathrm{pin}}^{\mathrm{liq}}$, it is necessary to take into account that the displacements generated by the dislocation network are essentially larger than the displacements existing in the Bragg glass at the same temperature and magnetic field within the scales $l_{\perp}, l_{\|}$. Indeed, in the lattice without dislocations, one has $u\left(l_{\perp}, 0\right) \sim u\left(0, l_{\|}\right)=u<\xi$ [see Eq. (29)], while in the liquid the dislocations lead to displacements of about $a>\xi$ at the boundary of the dislocation cell. It is also essential that the displacements caused by the dislocations within a scale $l<l_{\perp}, l_{\|}$have a different dependence on $l$ than the elastic displacements in the Bragg glass. Then, we obtain the estimate:

$$
E_{\text {pin }}^{\text {liq }} \sim\left(W l_{\|} l_{\perp}^{2}\right)^{1 / 2} \xi
$$

Note that although the displacements generated by the dislocations are large and exceed $\xi$, we multiply the mean pinning force per cell only by $\xi$ in formula (30) since $\xi$ is the effective range of the elementary pinning force $f_{\text {pin }}$. It follows from Eqs. (28)-(30) that $E_{\text {pin }}^{\text {liq }} / E_{\text {pin }}^{\text {lat }} \sim(\xi / u) \sim$ $\left[1+\ln \left(R_{c}^{2} / a^{2}\right)\right]^{1 / 2}$, i.e., $E_{\text {pin }}^{\text {liq }}$ noticeably exceeds $E_{\text {pin }}^{\text {lat }}$ in the bundle pinning regime, and hence formula (30) gives the estimate of $E_{\text {pin }}$ in Eq. (27).

It is convenient to rewrite expression (30) in terms of $L_{c}$, the single-vortex collective pinning length, using the relation, ${ }^{9} f_{\text {pin }}^{2} n \approx \epsilon^{4} \varepsilon_{0}^{2} / L_{c}^{3}$. Then one arrives at the formula for $E_{\mathrm{pin}}$ :

$$
E_{\text {pin }} \sim \epsilon \varepsilon_{0} a\left[D g_{0}(t)\right]^{3 / 2} h^{1 / 4}(1-h)^{3 / 4},
$$

where we have inserted the estimates for $l_{\perp}, l_{\|}$, have taken into account that the quantity $\varepsilon_{0} \propto \lambda_{a b}^{-2}$ should lead to an additional factor $1-h$ when $h=H / H_{c 2}(t)$

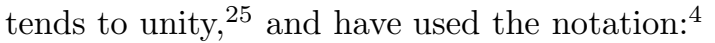

$$
\frac{\epsilon \xi(t)}{L_{c}(t)} \equiv D g_{0}(t)
$$

with $\epsilon \xi(0) / L_{c}(0)=D$. The function $g_{0}(t)$ is given ${ }^{4}$ by

$$
g_{0}(t)=\left(1-t^{2}\right)^{1 / 2}
$$

for $\delta l$ pinning, and by

$$
g_{0}(t)=\left(1-t^{2}\right)^{-1 / 6}
$$

for $\delta T_{c}$ pinning. The parameter $D$ is a measure of the pinning strength and is estimated as $9 \approx\left(j_{c} / j_{0}\right)^{1 / 2}$ where $j_{c}$ is the critical current density in the single vortex pinning regime and $j_{0}$ is the depairing current density, both taken at $T=0$.

Let us now estimate the entropy term in the energy balance (27). In Sec. II B we obtained the expressions for the melting line of the ideal vortex lattice, assuming that the main contribution to the entropy gain at melting is due to disappearance of the shear phonon modes in the liquid. The mechanism of this disappearance is the following: A shear stress in the liquid generates the socalled Peach-Köhler forces ${ }^{40.41}$ exerted on dislocations; the dislocations begin to move, and their shifts relax the shear stress in the liquid. In the vortex system with pinning a dislocation cannot move if the pinning force per dislocation cell, $\left(W l_{\|} l_{\perp}^{2}\right)^{1 / 2}$, exceeds the Peach-Köhler force $a c_{66} u_{x y} l_{\|}$exerted on a dislocation segment of length $l_{\|}$where $u_{x y}$ is the shear deformation of the vortex system in the plane normal to the magnetic field. Thus, for relaxation of the shear stress to occur, the deformation $u_{x y}$ must exceed the critical value

$$
u_{x y}^{c r}=\frac{\left(W l_{\|} l_{\perp}^{2}\right)^{1 / 2}}{a c_{66} l_{\|}} \sim\left[D g_{0}(t)\right]^{3 / 2} h^{-1 / 4}(1-h)^{-3 / 4} .
$$

Here we again have inserted the relations used in deriving Eq. (31). In the volume with dimensions $L_{\perp}$ and $L_{\|}$, thermal fluctuations generate shear deformations $u_{x y}$ that can be estimated from

$$
c_{66} u_{x y}^{2} L_{\|} L_{\perp}^{2} \sim T .
$$


Hence, for large $L_{\perp}$ and $L_{\|}$, when

$$
L_{\|} L_{\perp}^{2}>\left[L_{\|} L_{\perp}^{2}\right]^{c r} \sim \frac{T}{c_{66}\left(u_{x y}^{c r}\right)^{2}}
$$

the deformations are less than the critical value; the relaxation of the shear stress in the liquid does not occur, and these modes do not contribute to the entropy gain (they do not differ essentially from the appropriate modes in the Bragg glass). Then, applying the considerations of Sec. II B, we obtain the factor $\Delta S$ in Eq. (27): $\Delta S=1-P(t, h)$, where

$$
P(t, h)=\frac{l_{\|} l_{\perp}^{2}}{\left[L_{\|} L_{\perp}^{2}\right]^{c r}} \sim \frac{\left[D g_{0}(t)\right]^{3}\left(1-t^{2}\right)^{1 / 2}}{t G i^{1 / 2} h} .
$$

As is well known,, 9.39 thermal fluctuations of the fluxline lattice lead to a smoothing of the pinning potential and thereby affect the pinning. We take into account the effect of this thermal depinning on $E_{\text {pin }}$ and $P(t, h)$ using the recipe of Ref. 9 (see page 1214 in that paper). Then an additional factor $\xi / r_{p}$ appears in Eq. (30), while the right hand side of Eq. (35) is multiplied by $\left(\xi / r_{p}\right)^{2}$ where $r_{p}=\left(\xi^{2}+u_{T}^{2}\right)^{1 / 2}$ is the new effective range of the pinning force when thermal fluctuations are allowed for. This modifies formulas (31) and (37) as follows:

$$
\begin{gathered}
E_{\text {pin }} \sim \epsilon \varepsilon_{0} a \frac{\left[D g_{0}(t)\right]^{3 / 2} h^{1 / 4}(1-h)^{3 / 4}}{\left[1+t\left(\frac{G i}{1-t^{2}}\right)^{1 / 2} \frac{f(h)}{h^{1 / 2}}\right]^{1 / 2}}, \\
P(t, h) \sim \frac{\left[D g_{0}(t)\right]^{3}\left(1-t^{2}\right)^{1 / 2}}{t G i^{1 / 2} h\left[1+t\left(\frac{G i}{1-t^{2}}\right)^{1 / 2} \frac{f(h)}{h^{1 / 2}}\right]^{2}},
\end{gathered}
$$

where we have used Eq. (6) for $u_{T}^{2}$.

Inserting formulas (38) in Eq. (27), expressing $C$ as $8 \sqrt{\pi} c_{L}^{2} / f_{1}$ with a constant $f_{1}$, and taking into account that $\Delta F=0$ at melting, we eventually find the equation for the melting line $h_{m}(t)$ :

$$
\begin{gathered}
\frac{F_{T}(t) h_{m}^{1 / 2}}{\left(1-h_{m}\right)^{3 / 2}}\left[1-P\left(t, h_{m}\right)\right]+ \\
\frac{A\left[D g_{0}(t)\right]^{3 / 2} h_{m}^{1 / 4}}{\left[\left(1-h_{m}\right)^{3 / 2}+F_{T}(t) h_{m}^{-1 / 2}\right]^{1 / 2}}=2 \pi c_{L}^{2},
\end{gathered}
$$

where

$$
P(t, h)=\frac{B\left[D g_{0}(t)\right]^{3}\left(1-t^{2}\right)^{1 / 2}}{t G i^{1 / 2}\left[h^{1 / 2}+F_{T}(t)(1-h)^{-3 / 2}\right]^{2}},
$$

$A, B$ are some numerical factors, and we have used the notation

$$
F_{T}(t)=f_{1} t\left(\frac{G i}{1-t^{2}}\right)^{1 / 2}
$$

putting $f_{1}=2$ below. Equation (39) generalizes Eq. (20) obtained in the case of the ideal lattice. Note that the factor $1-P=\Delta S$ in Eq. (39) naturally explains the existence of the upper critical point on the melting line. The temperature $t_{\text {up }}$ of this point can be obtained from the condition $\Delta S=0$, or

$$
P\left(t_{\mathrm{up}}, h_{m}\left(t_{\mathrm{up}}\right)\right)=1,
$$

i.e., the entropy jump in the vortex system at melting vanishes at this point.

Equation (39) is in agreement with the general conclusion made in Ref. 4. Namely, it was stated ${ }^{4}$ that the phase diagrams of various three dimensional superconductors with point defects are determined only by the Ginzburg number $G i$, the parameter $D$ characterizing the strength of pinning, and the function $g_{0}(t)$ which is defined by the type of pinning. Other physical quantities [like $T_{c}, H_{c 2}(T), \epsilon$, etc.] either determine only scaling factors or do not appear explicitly in the appropriate equations at all. In particular, the anisotropy $\epsilon$ is absorbed by the definitions of $G i$ and $D$ and does not enter in Eq. (39).

\section{B. Analysis of the equation}

Figures 2 and 3 present the melting lines $H_{m}(t)$ obtained numerically from Eq. (39) for different pinning strength $D$. Although the numerical factors $A$ and $B$ have remained unknown in the above derivation of Eq. (39), the figures show that it is possible to choose $A_{1}=A /(2 \pi)^{3 / 4}$ and $B_{1}=B f_{1} /(2 \pi)^{3 / 2}$ so that the calculated melting lines have the properties observed in experiments (see Introduction). In particular, we find that the upper critical point noticeably shifts under the influence of pinning and tends to $T_{c}$ at reasonable values of $D$ when $A_{1}$ and $B_{1}$ are not too small $\left(A_{1}, \sqrt{B}_{1} \sim 1\right)$. With increasing $D$ the melting line shifts downwards if $B_{1}<A_{1}^{2}$, and this shift becomes small when $B_{1}$ does not differ too much from $A_{1}^{2}$. Of course, the choice of $A_{1}$ and $B_{1}$ on the basis of these requirements is not unique. Let us now analyze Eq. (39) in some limiting cases.

According to Sec. II, in the temperature region (12) one has $1-h_{m} \ll 1$ for the melting line of the ideal vortex lattice. If the parameter $D$ is not too large, this property of the melting line remains true for the vortex lattice with pinning. But under the condition $1-h_{m} \ll 1$ the lowest Landau level approximation is valid, and we can use the results of Appendix A where the effect of quenched disorder on the melting line has been analyzed in the framework of an approach similar to that of Sec. II C. On the other hand, putting $h_{m} \approx 1$ in Eq. (39), we arrive at

$$
\begin{aligned}
Q^{-3 / 2}\left(1-\frac{B f_{1}[G(t)]^{2}}{\left[1+Q^{-3 / 2}\right]^{2}}\right) & + \\
\frac{A G(t) Q^{-3 / 4}}{\left[1+Q^{-3 / 2}\right]^{1 / 2}} & =2 \pi c_{L}^{2},
\end{aligned}
$$


where $Q^{3 / 2}=\left(1-h_{m}\right)^{3 / 2} / F_{T}(t)$ [compare with formula [21)], and

$$
G(t)=\frac{\left[D g_{0}(t)\right]^{3 / 2}}{\left[F_{T}(t)\right]^{1 / 2}}=\frac{\left[D g_{0}(t)\right]^{3 / 2}\left(1-t^{2}\right)^{1 / 4}}{\left(f_{1} t\right)^{1 / 2} G i^{1 / 4}} .
$$

Equation (43) shows that the quantity $Q$ on the melting line depends on $t$ only via the function $G(t)$. This conclusion completely agrees with the exact result of Appendix A.

When $D=0$ [i.e., $G(t)=0$ ], Eq. (43) gives the result for the ideal vortex lattice, $Q^{-3 / 2}=2 \pi c_{L}^{2}$, see Eq. (13). For nonzero $D$ the function $G(t)$ decreases with the temperature. In the case of $\delta T_{c}$ pinning one has $G(t) \propto t^{-1 / 2}$, while $G(t)$ is proportional to $\left(1-t^{2}\right) / \sqrt{t}$ for $\delta l$ pinning. Hence in the equation for the melting line the role of terms associated with pinning increases with decreasing $t$. It follows from Eqs. (43), (42) that at the upper critical point the function $G(t)$ reaches a certain value, and condition (42) can be written in the form:

$$
\sqrt{B f_{1}} G\left(t_{\mathrm{up}}\right)=\frac{1}{2}+\left(\frac{1}{4}+\left(2 \pi c_{L}^{2}\right)^{2} \frac{B f_{1}}{A^{2}}\right)^{1 / 2} .
$$

Interestingly, up to a numerical factor (and the factor [1$\left.h_{s v}(t)\right]^{3 / 2}$ which we do not take into account here ${ }^{42}$ ) this condition reduces to Eq. (32) of Ref. 4 for the intersection point of the melting line with the order-disorder line. In particular, it follows from Eqs. (44), (45) that $t_{\text {up }}$, similarly to the temperature of the intersection point, depends on $G i$ and $D$ only via the combination $D^{3} / G i^{1 / 2}$ of these parameters.

The so-called depinning line $\frac{9}{}$ along which $u_{T} \approx \xi$, intersects the melting line of the ideal lattice, $H_{m}^{\mathrm{id}}(t)$, at $1-t^{2} \sim\left(f_{1}^{2} / 2 \pi c_{L}^{2}\right) G i$, i.e., outside the temperature region defined by Eq. (12). In the region $1-t^{2} \ll$ $\left(f_{1}^{2} / 2 \pi c_{L}^{2}\right) G i$, the depinning is essential. In this depinning region, the terms $T \Delta S$ and $E_{\text {pin }}$ in the energy balance (27) become relatively small, and the melting line is close to that of the ideal lattice.

\section{Lindemann criterion}

We now rewrite Eq. (39) in the form of the Lindemann criterion. As it follows from formulas (5) and (9), the factor $F_{T}(t) h_{m}^{1 / 2} /\left(1-h_{m}\right)^{3 / 2}$ in the first term of Eq. (39) is simply $2 \pi u_{T}^{2} / a^{2}$ where $u_{T}$ is described ${ }^{43}$ by Eq. (6). To rewrite the second term in Eq. (39), let us take into account the formula for the averaged relative shift of two line elements in the vortex lattice separated by a distance $R \leq R_{c}$ transverse to the magnetic field: $\underline{\underline{44}}$

$$
\frac{u^{2}(R, 0)}{r_{p}^{2}} \approx\left(\frac{\epsilon a}{L_{c}} \frac{\xi^{2}}{r_{p}^{2}}\right)^{3} \frac{1+\ln \left(R^{2} / a^{2}\right)+\epsilon R / \lambda_{a b}}{(1-h)^{3 / 2}} .
$$

[If the small bundle pinning regime occurs, $R_{c}<\lambda_{a b}$, the last term in this formula, $\epsilon R / \lambda_{a b}$, is small and may
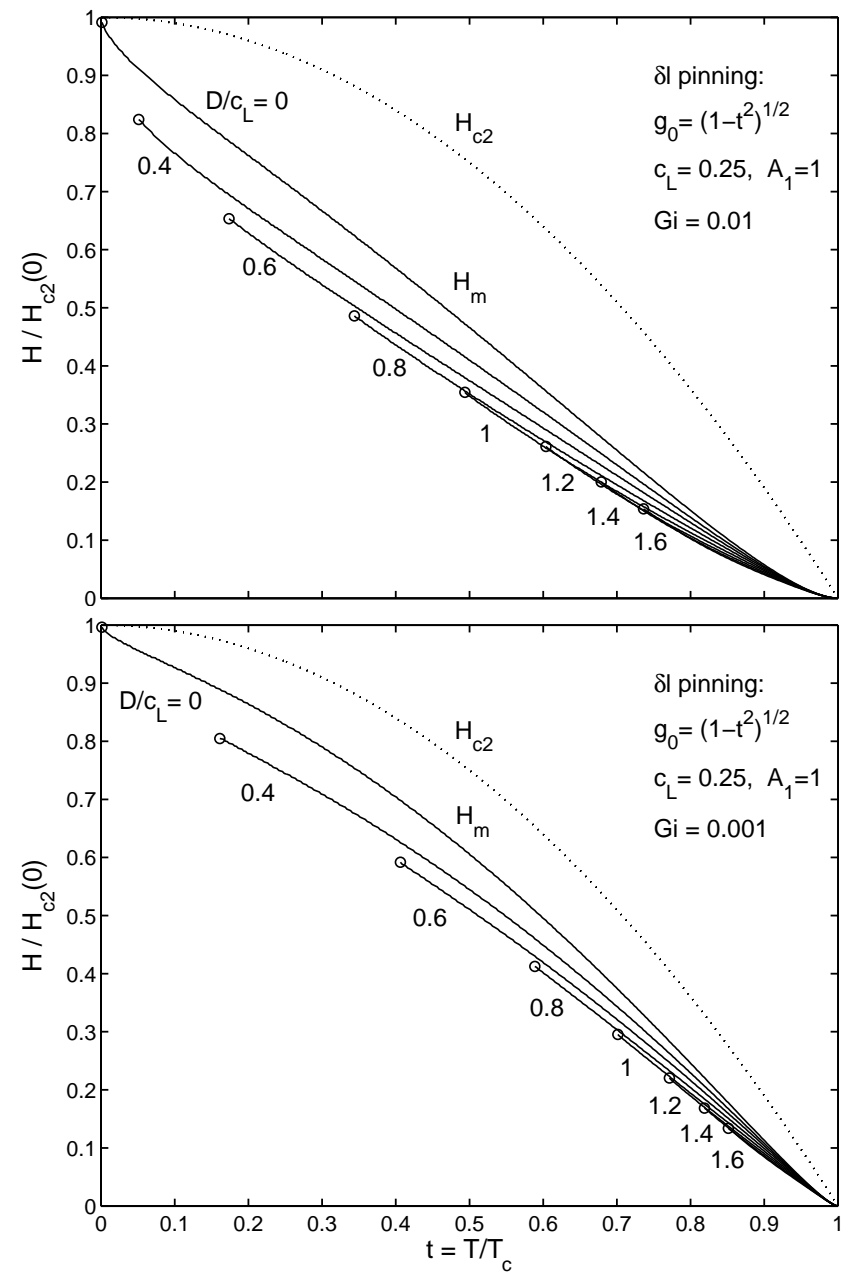

FIG. 2: The melting line $H_{m}(t)$ for the vortex lattice with quenched disorder, from Eq. (39), for $D / c_{L}=0,0.4,0.6,0.8$, $1,1.2,1.4,1.6$ in the case of $\delta l$ pinning at $G i=0.01$ (top) and at $G i=0.001$ (bottom). Here $A_{1}=A /(2 \pi)^{3 / 4}=1$. The upper line $D=0$ is the melting line of the ideal lattice, $H_{m}^{\mathrm{id}}(t)$. The dotted line shows $H_{c 2}(t) / H_{c 2}(0)=1-t^{2}$.

be omitted.] At $R=R_{c}$, one has $u\left(R_{c}, 0\right)=r_{p}$. Inserting $r_{p}^{2}=\xi^{2}+u_{T}^{2}$ and the expression (6) for $u_{T}$ in the relationship thus obtained, and taking into account the definition (32), we arrive at

$$
\begin{aligned}
1+\ln \left(\frac{R_{c}^{2}}{a^{2}}\right)+\epsilon \frac{R_{c}}{\lambda_{a b}} & =\left(\frac{h(1-h)}{2 \pi D^{2} g_{0}^{2}(t)}\right)^{3 / 2} \times \\
& {\left[1+\left(\frac{G i}{1-t^{2}}\right)^{1 / 2} \frac{f_{1} t}{h^{1 / 2}(1-h)^{3 / 2}}\right]^{3} . }
\end{aligned}
$$

Simple manipulations using this formula show that the second term in Eq. (39), which tells the role of the pinning energy in the energy balance, is

$$
2 \pi A_{1} \frac{r_{p}^{2}}{a^{2}}\left[1+\ln \left(\frac{R_{c}^{2}}{a^{2}}\right)+\frac{\epsilon R_{c}}{\lambda_{a b}}\right]^{-1 / 2},
$$

where $A_{1}=A /(2 \pi)^{3 / 4}$. Since the factor containing $R_{c}$ can be represented as $u(a, 0) / r_{p}$ [using Eq. (46) at $R=a$ 

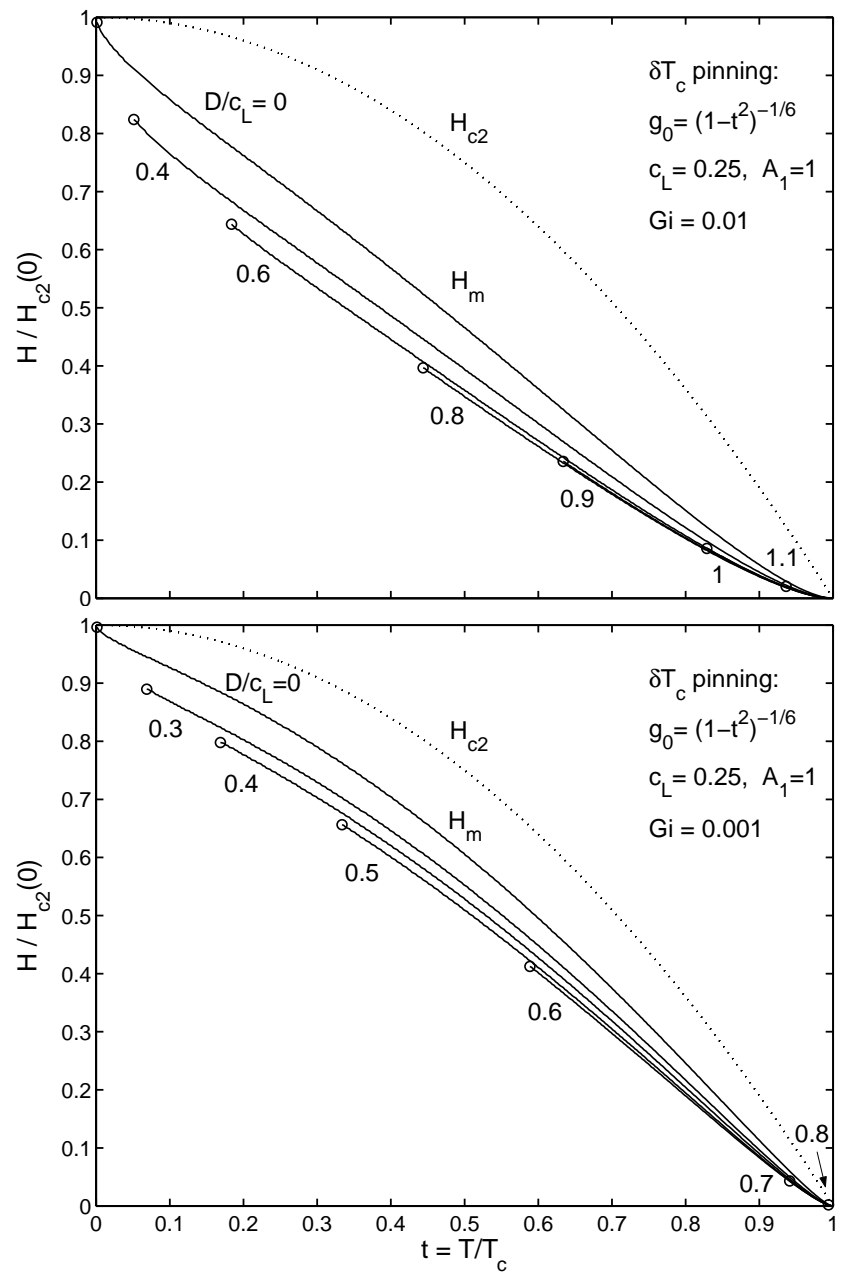

FIG. 3: As Fig. 2 but for $\delta T_{c}$ pinning.

and then at $R=R_{c}$ ], we find one more form for the second term in Eq. (39):

$$
2 \pi A_{1} \frac{r_{p} u(a, 0)}{a^{2}} .
$$

As to the quantity $P(t, h)$ given by Eq. (40), it is expressed as $B_{1} u^{2}(a, 0) / u_{T}^{2}$ where $B_{1}=B f_{1} /(2 \pi)^{3 / 2}$. Thus, equation (39) is equivalent to the following criterion:

$$
u_{T}^{2}-B_{1} u^{2}(a, 0)+A_{1} r_{p} u(a, 0)=c_{L}^{2} a^{2},
$$

which is true for the melting line in the bundle pinning region. Equation (50) can be also rewritten in the form:

$$
\begin{aligned}
u_{T}^{2}-B_{1} u^{2}(a, 0)+A_{1} u^{2}(a, 0)\left[1+\ln \left(\frac{R_{c}^{2}}{a^{2}}\right)\right. & \left.+\frac{\epsilon R_{c}}{\lambda_{a b}}\right]^{1 / 2} \\
& =c_{L}^{2} a^{2} .
\end{aligned}
$$

Note that although criteria (21) and (31) are qualitatively close to Eq. (51), they underestimate pinning at large $R_{c}$.

Equation (50) [or (51)] is valid when $u_{T}^{2} \geq B_{1} u^{2}(a, 0)$ since condition (42) for the upper critical point now has the form:

$$
u_{T}^{2}=B_{1} u^{2}(a, 0) .
$$

Taking into account that $B_{1} \sim 1$, this condition means that the upper critical point, as a rule, lies in the bundle pinning region. Indeed, if the melting line enters the single vortex pinning region before the upper critical point occurs, one finds $u_{T}^{2} \geq B_{1} r_{p}^{2}=B_{1}\left(\xi^{2}+u_{T}^{2}\right)$ at the boundary of this region. In other words, the melting line can intersect the boundary in the depinning region where $u_{T}^{2}$ is large. But in this region the melting line practically coincides with that of the ideal lattice, Sec. III B; the latter can cross the boundary only in the case of $\delta T_{c}$ pinning at sufficiently large $D .19$

\section{PHASE DIAGRAMS}

\section{A. The first and the second scenarios}

To gain some insight into the character of the mergence of the melting line with the order-disorder line, let us consider more closely the dependence of the free energy difference between the liquid and the Bragg glass, $\Delta F$, on the size of the dislocation cell, $l_{\perp}$. We shall consider only such $l_{\perp}$ that lie near the minimum possible size in the lattice $l_{\perp}^{\min }\left(l_{\perp}^{\mathrm{min}} \sim a\right)$. In evaluating the entropy gain $T \Delta S$, the elastic energy $E_{\mathrm{el}}$ and the pinning energy $E_{\mathrm{pin}}$, it is necessary to take into account that the modulus $c_{44}$ depends on the wave vector $\mathbf{k}$. According to Ref. 25, one has $c_{44}\left(k \sim 1 / l_{\perp}\right) \approx c_{44}(1 / a) \cdot\left(l_{\perp} / a\right)^{2}$ at $l_{\perp}<\lambda_{a b} / \epsilon$, and hence $l_{\|} \propto l_{\perp}^{2}$, see formula (17). Then we can write $\Delta F$ in the form (we still neglect logarithmic factors):

$$
\Delta F \propto E_{\mathrm{el}}(a) \tilde{l}_{\perp}^{2}-E_{\mathrm{pin}}(a) \tilde{l}_{\perp}^{2}+T\left[P(a) \tilde{l}_{\perp}^{4}-1\right],
$$

where $\tilde{l}_{\perp}=l_{\perp} / l_{\perp}^{\mathrm{min}}, P(a)$ denotes $P\left(t, h_{m}\right)$ of Eq. (39), and $E_{\mathrm{el}}(a)$ and $E_{\mathrm{pin}}(a)$ are the elastic and the pinning energies per dislocation cell at $l_{\perp}=l_{\perp}^{\mathrm{min}}$, which have been inserted into formula (27) to derive Eq. (39). Equation (53) is valid when $P(a) \tilde{l}_{\perp}^{4}-1<0$, otherwise the last term in this equation has to be omitted, and one has,

$$
\Delta F \propto E_{\mathrm{el}}(a) \tilde{l}_{\perp}^{2}-E_{\mathrm{pin}}(a) \tilde{l}_{\perp}^{2},
$$

for $\tilde{l}_{\perp}^{4}>1 / P(a)$.

As was mentioned above, in the equation (39) for $H_{m}(t)$ [or equivalently $T_{m}(H)$ ] the relative role of terms associated with pinning increases with decreasing $T_{m}$ for both types of pinning. When $E_{\text {pin }}(a)$ and $P(a)$ are sufficiently small (i.e., for temperatures $T_{m}$ near $T_{c}$ ), the function $\Delta F\left(\tilde{l}_{\perp}\right)$ is minimum at the lowest possible value $\tilde{l}_{\perp}=$ 1 and then increases with increasing $\tilde{l}_{\perp}$, Fig. 迎. This means that melting occurs at $\tilde{l}_{\perp}=1$ as in the ideal lattice. This conclusion has been already used implicitly in deriving Eq. (39). When the temperature $T_{m}$ decreases, and hence $P(a)$ and the ratio $E_{\mathrm{pin}}(a) / E_{\mathrm{el}}(a)$ increase, the 
point $\tilde{l}_{\perp}=[1 / P(a)]^{1 / 4}$ shifts to smaller $\tilde{l}_{\perp}$, and the slope of $\Delta F$ in Eq. (54) on $\tilde{l}_{\perp}^{2}$ decreases. Eventually, at the critical temperature $t_{\mathrm{up}}$, one arrives at the situation shown either in Fig. 4: or in Fig. 4 $\mathrm{d}$ since the energy balance for the melting $E_{\mathrm{el}}(a)-E_{\mathrm{pin}}(a)=T_{m}[1-P(a)]$ leads to $E_{\mathrm{el}}(a)-E_{\mathrm{pin}}(a)=0$ at this point. In the case of Fig. [4 when the dependence of $\Delta F$ on $\tilde{l}_{\perp}^{2}$ has a negative curvature at $\tilde{l}_{\perp}=1$, we conclude that this case is necessarily preceded by the situation shown in Fig. $4 \mathrm{~b}$, which has to occur at some temperature $t_{i}>t_{\text {up }}$ where $P(a)<1$ and $E_{\text {pin }}(a)<E_{\text {el }}(a)$. At this $t_{i}$ the size of the dislocation cell, $l_{\perp}$, sharply increases because the absolute minimum of $\Delta F$ now occurs at a larger $\tilde{l}_{\perp}>1$. In other words, at this temperature we find an intersection of the melting line with the order-disorder line. The abrupt increase of $l_{\perp}$ also means that the melting and the order-disorder transition cannot form a unified phase transition line. Thus, we arrive at the first scenario $\frac{45}{5}$ shown in Fig. [1] In the case of Fig. 枯 two possibilities exist: Either at some temperature $t_{i}>t_{\mathrm{up}}$ the situation shown in Fig. 40 occurs, and we again arrive at the first scenario, or the function $\Delta F\left(\tilde{l}_{\perp}\right)-\Delta F(1)$ remains positive for all $\tilde{l}_{\perp}$ in the temperature interval $t_{\text {up }} \leq t \leq 1$. In the latter case we find that the mean size of the dislocation cell, $l_{\perp}$, cannot gradually change in the process of the reduction of the entropy gain, and hence one has $\tilde{l}_{\perp}=1$ down to $t_{\mathrm{up}}$. In this case, the melting line continuously transforms into the order-disorder line, and we arrive at the second scenario. Moreover, since $t_{\mathrm{up}}$ is not a specific temperature for $E_{\mathrm{pin}}(a)$ or $E_{\mathrm{el}}(a)$, it is quite probable that the result $\tilde{l}_{\perp}=1$ remains true also at $t<t_{\text {up }}$ (at least in the bundle pinning region). Then, at $t \leq t_{\text {up }}$ the order-disorder line (which is now a part of the unified phase transition line) is determined by the condition,

$$
E_{\mathrm{el}}(a)=E_{\mathrm{pin}}(a)
$$

As it follows from the results of Sec. III C, in the bundle pinning region condition (55) is equivalent to the Lindemann criterion:

$$
A_{1} u^{2}(a, 0)\left[1+\ln \left(\frac{R_{c}^{2}}{a^{2}}\right)+\frac{\epsilon R_{c}}{\lambda_{a b}}\right]^{1 / 2}=c_{L}^{2} a^{2} .
$$

If the order-disorder line enters the single vortex pinning region, where $R_{c}=a$, condition (55) yields:

$$
A_{1} u^{2}(a, 0)=c_{L}^{2} a^{2} .
$$

(Note that the ratio $\epsilon a / \lambda_{a b}$ is negligible.) In Appendix $\mathrm{B}$ equations (56) and (57) are presented in explicit form.

To calculate the order-disorder line $H_{d i s}(t)$ in the case of the first scenario, we used the criterion $\underline{\underline{4}}$

$$
u^{2}(a, 0)=c_{L D}^{2} a^{2},
$$

where $c_{L D}$ is some new Lindemann constant describing the order-disorder transition. It should be emphasized that although Eq. (58) is similar to Eq. (57), the Lindemann constant $c_{L D}$ in Eq. (58) is independent of the

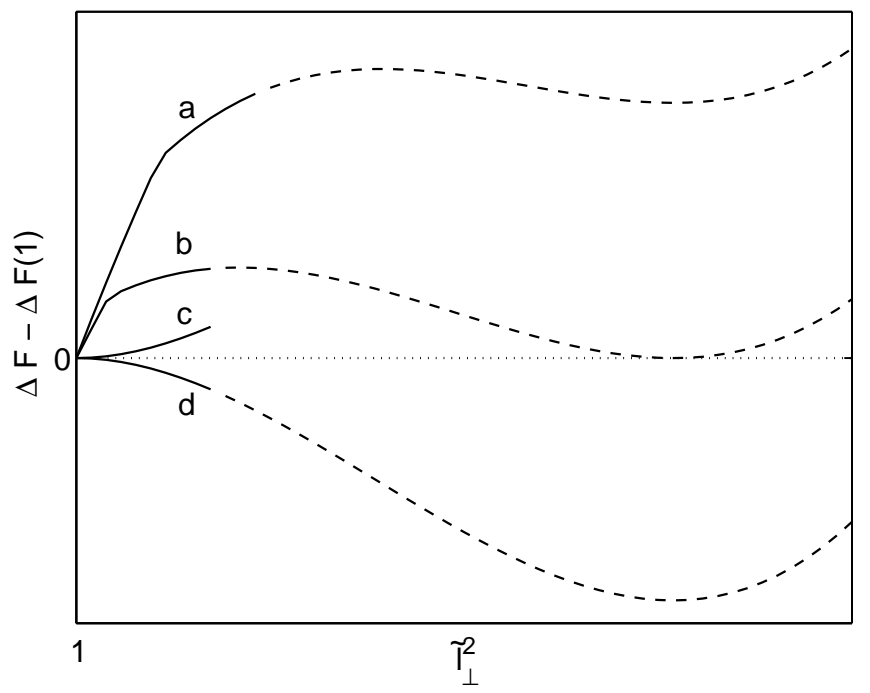

FIG. 4: Schematic sketch of the function $\Delta F\left(\tilde{l}_{\perp}\right)$, Eqs. (53), (54), for $t>t_{i}$ (a), $t=t_{i}$ (b), and $t=t_{\mathrm{up}}$ (c or d). The parts of the curves where Eqs. (53), (54) fail are indicated as dashed lines. The breaks of the curves $\mathrm{a}$ and $\mathrm{b}$ occur at the points $\tilde{l}_{\perp}^{4}=1 / P(a)$ where Eq. (53) transforms into Eq. (54).

constant $c_{L}$ defining the melting, while in the case of the second scenario Eq. (57) leads to a relationship between the Lindemann constants defining the melting and the order-disorder lines (the ratio of these constants is $\sqrt{A}_{1}$ ). Criterion (58) both in the single vortex and in the bundle pinning ${ }^{46}$ regions were analyzed in Ref. 4, allowing for a smoothing of the pinning potential by thermal fluctuations. The appropriate equations in the explicit form are presented in Appendix B. For definiteness, in the analysis below we shall choose the constant $c_{L D}$ for the order-disorder line as equal to the constant $c_{L}$ for the melting line.

\section{B. Numerical results}

Although in this paper we have not analyzed the orderdisorder line in detail, criteria (56), (57), and (58) enable one to evaluate the location of this line in the $T-H$ plane for the first and the second scenarios. In Figs. 囵8 we compare the $T$ - $H$ phase diagrams of type-II superconductors with different types of pinning for these two scenarios. In the construction of the figures, we use the values of the Ginzburg number $G i=0.01,0.0001$ which are typical for high- $T_{c}$ superconductors, and we take into account the factor containing $\left[1-h_{s v}(t)\right]$ that has been omitted in Sec. III ${ }^{42}$ The complete set of the appropriate equations is given in Appendix B.

In Figs. [5 - 8 we choose the constants $A_{1}$ and $B_{1}$ so that the following two requirements are satisfied: First, the properties of $H_{m}(t)$ observed in experiments are reproduced, namely, with increasing $D$ the upper critical point clearly shifts, while the downward shift of the melt- 
ing line is small, see Sec. III B. Second, the upper critical point $t_{\mathrm{up}}$ of the melting line coincides with the intersection point $t_{i}$ of this line with the order-disorder line (calculated within the first scenario) at $D=0.7$ for the case of $\delta l$ pinning. The latter requirement is due to the following considerations: According to Ref. 12, 15, the coincidence of $t_{i}$ and $t_{\mathrm{up}}$ is observed in overdoped $\mathrm{YBa}_{2} \mathrm{Cu}_{3} \mathrm{O}_{y}$ crystals $(y>6.92)$ for which the upper critical point lies at sufficiently large magnetic fields, and so the case $h_{m} \sim 1$ appears to occur there. As it was mentioned in Sec. III B, in this case $t_{i}$ and $t_{\text {up }}$ depend on $G i$ and $D$ only via a single combination of these parameters, $D^{3} / G i^{1 / 2}$. Therefore, one may expect that if the coincidence of the upper critical point with the intersection point occurs, it practically will not depend on the specific choice of $D$ or $G i$. In other words, the coincidence will approximately occur for different $D, G i$, and types of pinning as long as $h_{i} \approx 1$. Although $h_{i}$ is not too close to unity in Figs. [5-17(top), the data of these figures support this statement. As one might expect, the region of the coincidence is especially wide in $D$ for small $G i$, Fig. 6] Thus, we have introduced the second requirement here in order to fit the phase diagrams calculated in the framework of the first scenario to the experimental situation observed in the overdoped $\mathrm{YBa}_{2} \mathrm{Cu}_{3} \mathrm{O}_{y}$ crystals.

The presented figures show that when the intersection point is close to the upper critical point, both scenarios lead to qualitatively similar phase diagrams. However, when the strength of pinning $D$ increases, and the intersection point shifts toward $T_{c}$, the coincidence of the points fails especially for $\delta T_{c}$ pinning. In this case the first scenario leads to a noticeable extension of the melting line beyond the intersection point. Thus, if the first scenario really occurs, this result possibly explains the experimental findings 11.12 .13 .14 .15 for optimally doped $\mathrm{YBa}_{2} \mathrm{Cu}_{3} \mathrm{O}_{y}$ crystals $(y \approx 6.92)$ in which the upper critical point lies at larger magnetic field than the intersection point. Note also that in the case of $\delta T_{c}$ pinning, the melting line enters the single vortex pinning region near $T_{c}$ at $\nu \equiv(2 \pi)^{3 / 2} D^{3} / G i^{1 / 2}$ of the order of several units ${ }^{4.19}$

In Figs. [5- 8 we also show the boundary of the single vortex pinning region, $H_{s v}(t)$. It should be noted that apart from the well-known lower single vortex pinning region, $\frac{9}{=}$ an upper region exists where this pinning occurs. The upper region of single-vortex pinning was discussed by Larkin and Ovchinnikov $\underline{47}$ in the context of the origin of the peak effect in low- $T_{c}$ superconductors. Without account of thermal fluctuations, this region adjoins to the $H_{c 2}(t)$ line and is caused by the softening of the vortex lattice near $H_{c 2}(t)$. However, this softening also leads to an increase of $u_{T}$, which reduces the strength of pinning. As a result of these two opposite tendencies, the upper region does not extend to $T_{c}$ and has the shape of a "tongue" 4 Interestingly, when the strength of pinning increases, the upper and lower single-vortex pinning regions can merge at low temperatures. This merging occurs for $D \geq(4 \pi)^{-1 / 2}$; this value is independent of $G i$ and the type of pinning since the merging starts at

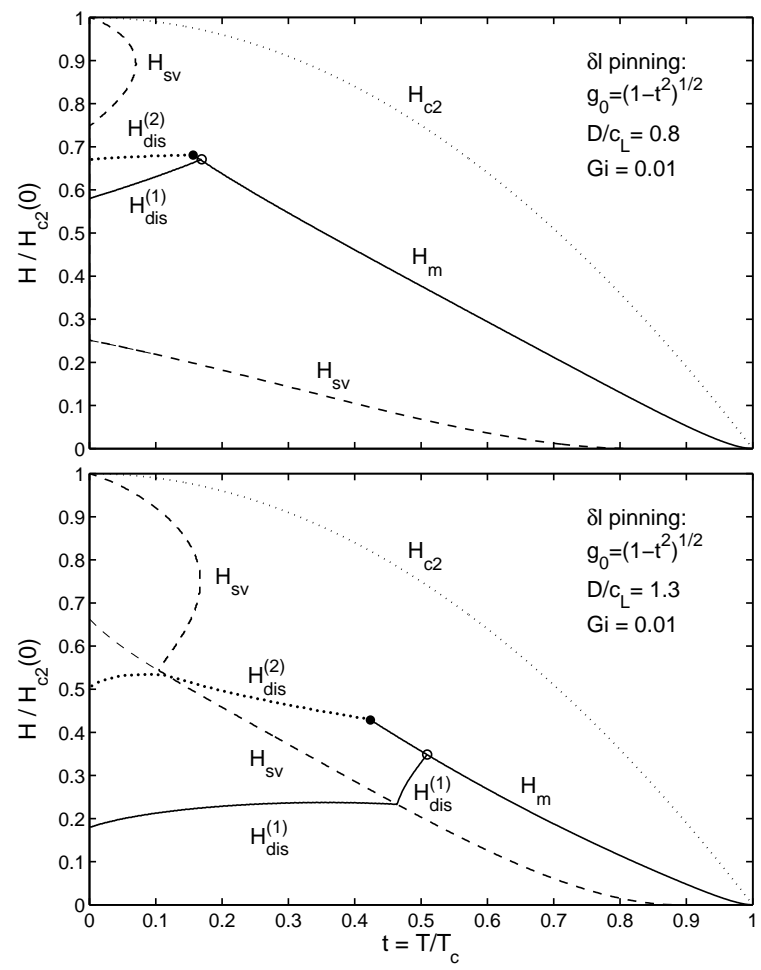

FIG. 5: The phase diagram in the case of $\delta l$ pinning, $g_{0}(t)=$ $\left(1-t^{2}\right)^{1 / 2}$. Here $A_{1}=0.66, B=0.8 A_{1}^{2}, c_{L}=0.25, G i=0.01$, and $D / c_{L}=0.8$ (top) or $D / c_{L}=1.3$ (bottom). The melting line $H_{m}(t)$ and the order-disorder line $H_{d i s}^{(1)}(t)$ for the first scenario are shown by solid lines, while the thick dotted line gives the order-disorder line $H_{d i s}^{(2)}(t)$ for the second scenario. The dashed line depicts the boundary of the single vortex pinning region, $H_{s v}(t)$, Eq. (B2) [the thin-dashed line shows Eq. (B1)]. The dotted line is $H_{c 2}(t) / H_{c 2}(0)=1-t^{2}$. The upper critical point $\left(T_{\mathrm{up}}, H_{\mathrm{up}}\right)$ is marked by a dot and the intersection point $\left(T_{i}, H_{i}\right)$ by a circle.

$T=0$.

The authors of Ref. 5 argued that only the second scenario can explain the decrease of $H_{d i s}$ with the temperature that was observed in their experiments. However, the presented figures show that the order-disorder line found from criterion (58) (the first scenario) can decrease or increase with temperature and even can be nonmonotonic. This depends on whether the line is in the single vortex pinning region or in the bundle pinning region and also on the Ginzburg number. It should be noted that in contrast to Eq. (3) we take into account only the displacement caused by the quenched disorder and do not include $u_{T}$ explicitly in the equation for the orderdisorder line, compare Eq. (58) with Eq. (37). However, the thermal depinning (which depends on $u_{T}$ ), the softening of the elastic moduli, and the possibility that the order-disorder line lies not only in the single vortex pinning region but also outside it, already produce the depicted variety of shapes of $H_{d i s}(t)$. Hence, even in the framework of the first scenario the presented results can explain the fact ${ }^{5}$ that the order-disorder lines observed 


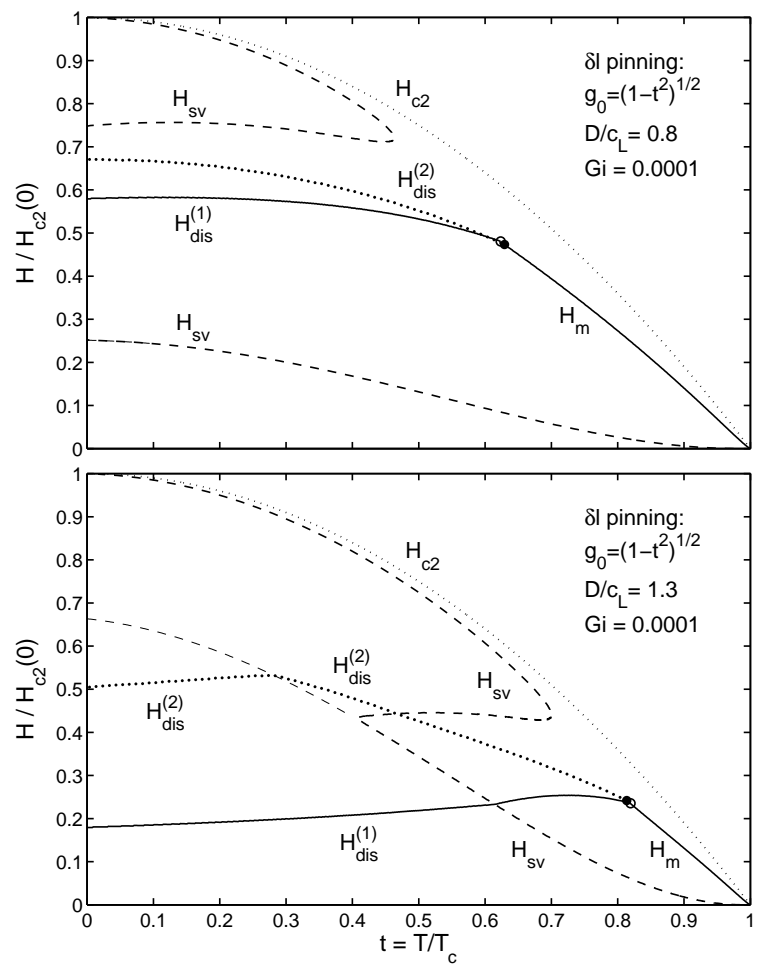

FIG. 6: As Fig. 5 but for $G i=0.0001$.
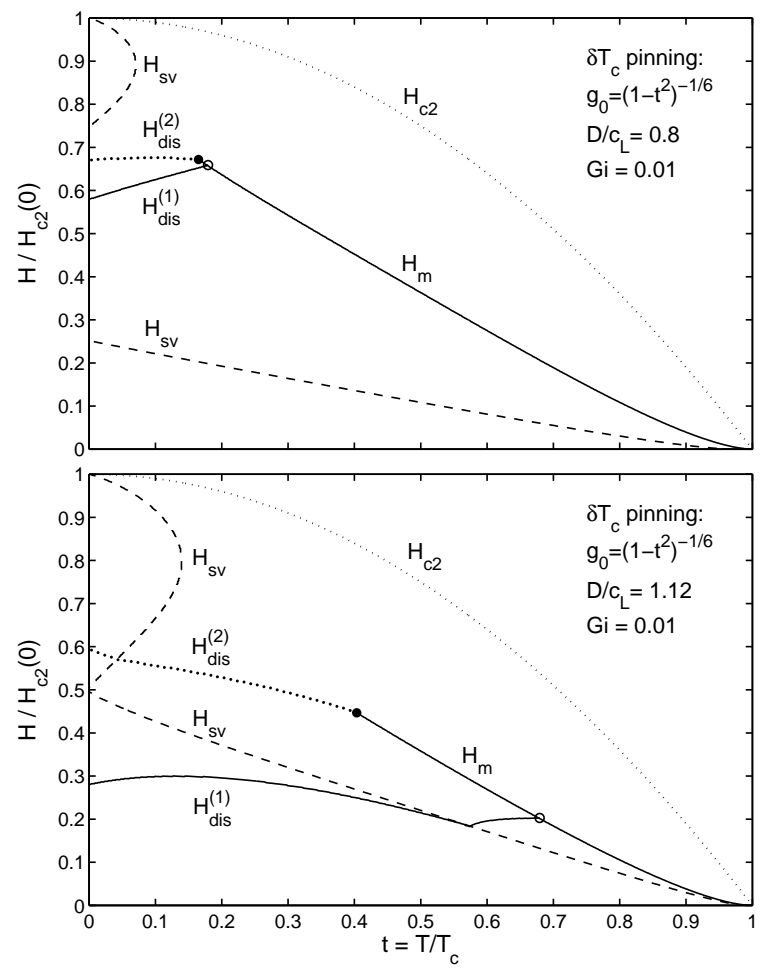

FIG. 7: As Fig. 5 but for the case of $\delta T_{c}$ pinning, $g_{0}(t)=$ $\left(1-t^{2}\right)^{-1 / 6}$. Top: $D / c_{L}=0.8$; bottom: $D / c_{L}=1.12$.
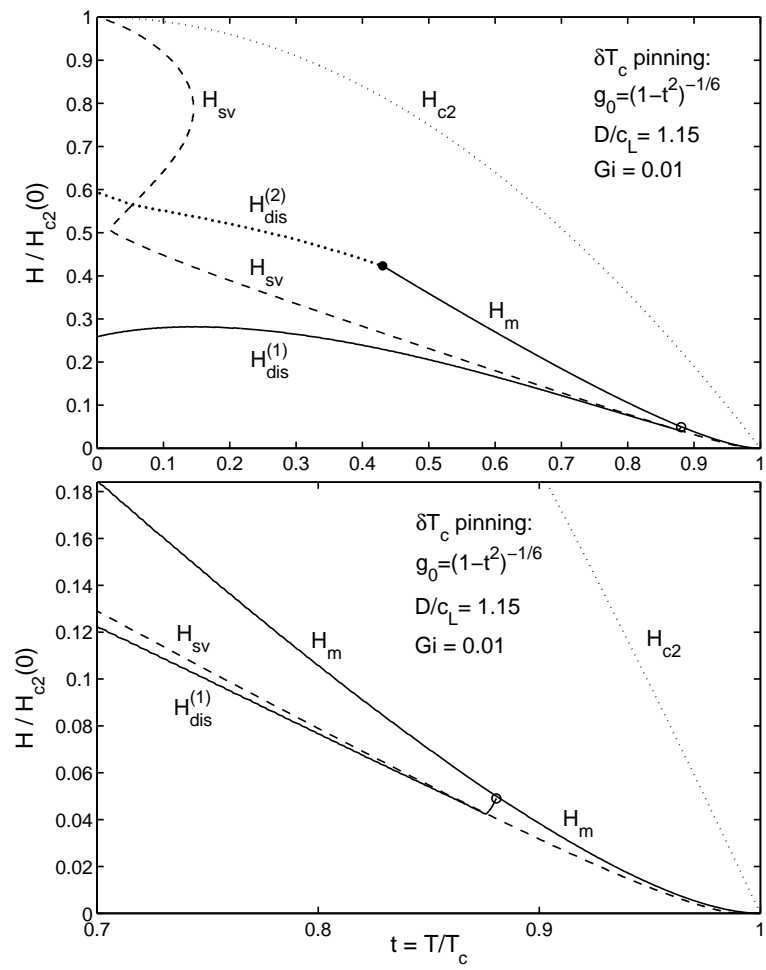

FIG. 8: As Fig. [7 but for $D / c_{L}=1.15$. The lower panel shows the same phase diagram near $T_{c}$ at enlarged scale.

in experiments have various shapes.

Finally, we briefly discuss the case of low- $T_{c}$ superconductors, which have a very small Ginzburg number. In this case one has $D^{3} / G i^{1 / 2} \gg 1$ even for weak pinning strength $D$. Since the temperatures $T_{i}$ and $T_{\text {up }}$ are mainly determined by this ratio of the parameters and increase when the ratio increases, we find that in low$T_{c}$ superconductors these temperatures practically coincide with $T_{c}$, and only the order-disorder line can be observed $\stackrel{48}{ }$ Interestingly, in this case the order-disorder lines for the first and for the second scenarios have the same functional dependences on $t$ and on the parameters $D, G i$ [this follows from equations (B5) and (B8)], and moreover, they practically coincide with each other when $A_{1}^{2} \approx 2 \pi c_{L}^{2}$.

\section{Acknowledgments}

This work was supported by the German Israeli Research Grant Agreement (GIF) No G-705-50.14/01.

\section{APPENDIX A: EFFECT OF DISORDER ON THE MELTING LINE: ANALYSIS BEYOND PERTURBATION THEORY.}

Assuming $1-t \ll 1$, we shall consider the partition function of the vortex system as a functional integral with 
the Ginzburg-Landau Hamiltonian ${ }^{30.34}$ In dimensionless units this Hamiltonian has the form:

$$
\begin{array}{r}
\frac{H_{G L}}{T}=\int d \mathbf{r}\left[\left|\partial_{z} \psi\right|^{2}+|(-i \nabla+\mathbf{A}) \psi|^{2}+\tau|\psi|^{2}+\right. \\
\left.2^{3 / 2} \pi G i^{1 / 2} t|\psi|^{4}\right]
\end{array}
$$

where $\psi$ is the order parameter; $\tau=t-1$; the coordinates $x$ and $y$ are measured in units of $\xi_{0}$, and $z$ in units of $\epsilon \xi_{0} ; \xi_{0}$ is the zero-temperature coherence length in the Ginzburg-Landau theory; the magnetic field is $b=$ $2 \pi \xi_{0}^{2} H / \Phi_{0}$ and $\mathbf{A}$ is its vector potential; $\nabla=\left(\partial_{x}, \partial_{y}\right)$. Consider the melting line $h_{m}(t)$ in the temperature region described by Eq. (12) in which $1-h_{m} \ll 1$, and hence the lowest Landau level approximation is valid (see Sec. II C). In this approximation the second term of Eq. (A1) reduces to

$$
b|\psi|^{2} .
$$

Pinning is introduced into the dimensional Ginzburg Landau Hamiltonian either via spatial disorder in the transition temperature $T_{c}+\delta T_{c}(\mathbf{r})\left(\delta T_{c}\right.$ pinning) or by spatial variation of the effective mass $m+\delta m(\mathbf{r})$ describing disorder in the mean free path $l$ of quasiparticles $(\delta l$ pinning) $\stackrel{9}{9}$ Here $m$ is the effective mass in the $x-y$ plane (since the magnetic field is along the $z$ axis). Thus, with quenched disorder in the vortex lattice, one should add to the dimensionless Hamiltonian (A1) the term

$$
\varphi(\mathbf{r})|\psi|^{2},
$$

where

$$
\varphi(\mathbf{r})=\frac{\delta T_{c}(\mathbf{r})}{T_{c}}
$$

in the case of $\delta T_{c}$ pinning, and

$$
\varphi(\mathbf{r})=\frac{\delta m(\mathbf{r})}{m} b \approx \frac{\delta m(\mathbf{r})}{m}|\tau|
$$

in the case of $\delta l$ pinning. In Eq. A5 we have used the relation

$$
b=|\tau| h
$$

that follows from the definitions of $b$ and $h$ and put $h=1$ since $1-h \ll 1$ in the lowest Landau level approximation. For pinning by point defects, it is assumed in the collective pinning theory ${ }^{9}$ that disorder in $\delta T_{c}(\mathbf{r})$ and in $\delta m(\mathbf{r})$ is short scale and described by a Gaussian distribution with zero mean value, $\left\langle\delta T_{c}(\mathbf{r})\right\rangle=\langle\delta m(\mathbf{r})\rangle=0$, and with the correlation function 49

$$
\frac{\left\langle\delta T_{c}(\mathbf{r}) \delta T_{c}\left(\mathbf{r}^{\prime}\right)\right\rangle}{T_{c}^{2}}=2 \pi D^{3} \delta\left(\mathbf{r}-\mathbf{r}^{\prime}\right)
$$

for $\delta T_{c}$ pinning, and

$$
\frac{\left\langle\delta m(\mathbf{r}) \delta m\left(\mathbf{r}^{\prime}\right)\right\rangle}{m^{2}}=\frac{30 \pi}{7} D^{3} \delta\left(\mathbf{r}-\mathbf{r}^{\prime}\right)
$$

for $\delta l$ pinning. Here $\langle\ldots\rangle$ means disorder averaging. Note that in agreement with Ref. 4 and with Sec. III A of this paper, equations (A1) - A8) again show that the phase diagrams of type-II superconductors with point defects depend only on $D$ and $G i$.

To proceed further, let us rescale the coordinates and the order parameter $\psi$ similarly to Ref. 35 :

$$
\begin{array}{r}
\tilde{x}=\sqrt{b} x, \\
\tilde{y}=\sqrt{b} y, \\
\tilde{z}=z G i^{1 / 6}(t b)^{1 / 3}, \\
\tilde{\psi}^{2}=\psi^{2} G i^{1 / 6} t^{1 / 3} b^{-2 / 3},
\end{array}
$$

where $\tilde{x}, \tilde{y}, \tilde{z}, \tilde{\psi}$ are the new coordinates and order parameter. Beside this, to agree with the notation used in the main text of this paper, we put $\tau=t^{2}-1$ below. Then, the Hamiltonian (A1) transforms into:

$$
\frac{H_{G L}}{T}=\int d \tilde{\mathbf{r}}\left[\left|\partial_{\tilde{z}} \tilde{\psi}\right|^{2}-Q|\tilde{\psi}|^{2}+2^{3 / 2} \pi|\tilde{\psi}|^{4}\right],
$$

where $Q$ is defined by Eq. (21). In the case of the ideal lattice, Eq. (23) for $h_{m}$ follows from this Hamiltonian, see Sec. II C. In a lattice with quenched disorder, the additional term from in the Hamiltonian has the form:

$$
\tilde{\varphi}(\tilde{\mathbf{r}})|\tilde{\psi}|^{2}
$$

with $\langle\tilde{\varphi}(\tilde{\mathbf{r}})\rangle=0$ and

$$
\left\langle\tilde{\varphi}(\tilde{\mathbf{r}}) \tilde{\varphi}\left(\tilde{\mathbf{r}}^{\prime}\right)\right\rangle=\frac{2 \pi D^{3}}{G i^{1 / 2} t} \delta\left(\tilde{\mathbf{r}}-\tilde{\mathbf{r}}^{\prime}\right)
$$

in the case of $\delta T_{c}$ pinning, and with

$$
\left\langle\tilde{\varphi}(\tilde{\mathbf{r}}) \tilde{\varphi}\left(\tilde{\mathbf{r}}^{\prime}\right)\right\rangle=\frac{30 \pi D^{3}\left(1-t^{2}\right)^{2}}{7 G i^{1 / 2} t} \delta\left(\tilde{\mathbf{r}}-\tilde{\mathbf{r}}^{\prime}\right)
$$

in the case of $\delta l$ pinning.

Up to numerical factors, the right hand sides of Eqs. A15) and (A16) coincide with the function $[G(t)]^{2}$ defined by Eq. (44). Then, the free energies of the vortex liquid, $F_{\text {liq }}$, and the vortex lattice, $F_{\text {lat }}$, are determined by $Q$ and $G(t)$, and along the melting line $h_{m}(t)$, one has:

$$
F_{\text {liq }}(Q, G(t))=F_{\text {lat }}(Q, G(t)) .
$$

Thus, on the melting line, the quantity $Q$ is some function of $G(t)$. Note that the functional form of Eq. (43) agrees with this conclusion, see Sec. III B.

\section{APPENDIX B: EQUATIONS FOR CALCULATION OF THE PHASE DIAGRAMS}

In section III where we considered the melting line in the bundle pinning region, the normalization factor containing $\left[1-h_{s v}(t)\right]$ was omitted ${ }^{42}$ The origin of this factor is due to the additional power of $1-h$ in the shear 
modulus $c_{66}$ as compared with the tilt modulus $c_{44}$, see Eqs. (16). In the single vortex pinning region where the shear modulus does not play any role, the additional power should not manifest itself in physical properties. Therefore, if a physical quantity in the bundle pinning region contains a factor $(1-h)^{n}$ caused by this additional power, it is necessary to introduce a normalization factor $\left[1-h_{s v}(t)\right]^{-n}$ in this quantity to provide its continuity at the boundary of the single vortex pinning region $h_{s v}(t)$. (Here $n$ is some power.) Although the effect of the factor $\left[1-h_{s v}(t)\right]^{-n}$ on the melting line $H_{m}(t)$ and on the order-disorder line $H_{d i s}(t)$ in the bundle pinning regime is small, it essentially influences the boundary of the single vortex pinning region, $H_{s v}(t)$, and the line $H_{d i s}(t)$ inside this region. In this Appendix B, taking into account this factor, 50 we compile the complete set of equations used for the construction of Figs. [5- 8

The boundary of the single vortex pinning region $h_{s v}(t)=H_{s v}(t) / H_{c 2}(t)$ is described by equation (19) of Ref. 4:

$$
h_{s v}^{1 / 2}(t)+\frac{F_{T}(t)}{\left[1-h_{s v}(t)\right]^{3 / 2}}=(2 \pi)^{1 / 2} D g_{0}(t),
$$

where we have used the notation (41). The boundary of the upper single vortex pinning region, discussed in Sec. IV B, can be obtained from the equation: $\underline{\underline{4}}$

$$
\frac{1-h}{1-h_{s v}(t)}\left[h^{1 / 2}+\frac{F_{T}(t)}{(1-h)^{3 / 2}}\right]^{2}=2 \pi\left(D g_{0}(t)\right)^{2},
$$

which in addition reproduces the root of Eq. (B1), i.e., it yields the entire boundary of the single vortex pinning regions. Inside this upper region the vortex lattice is in a state where $R_{c}=a$ and $u(a, 0)=r_{p}$, i.e., a borderline state between the single vortex pinning and bundle pinning regimes occurs there.

Equation (39) for the melting line in the bundle pinning region is rewritten as follows:

$$
\begin{gathered}
\frac{F_{T}(t) h_{m}^{1 / 2}}{\left(1-h_{m}\right)^{3 / 2}}\left[1-P\left(t, h_{m}\right)\right]+ \\
\frac{A\left[D g_{0}(t)\right]^{3 / 2} h_{m}^{1 / 4}\left[1-h_{s v}(t)\right]^{3 / 4}}{\left[\left(1-h_{m}\right)^{3 / 2}+F_{T}(t) h_{m}^{-1 / 2}\right]^{1 / 2}}=2 \pi c_{L}^{2}
\end{gathered}
$$

with

$$
P\left(t, h_{m}\right)=\frac{B\left[D g_{0}(t)\right]^{3}\left(1-t^{2}\right)^{1 / 2}\left[1-h_{s v}(t)\right]}{t G i^{1 / 2}\left[h_{m}^{1 / 2}+\frac{F_{T}(t)}{\left(1-h_{m}\right)^{3 / 2}}\right]^{2}} .
$$

Equation (B33) is valid in the interval $t_{\mathrm{up}} \leq t \leq 1$ where the temperature $t_{\mathrm{up}}$ defines the position of the upper critical point of the melting line and is given by the condition
(42). Note that in this paper we consider situations when the upper critical point lies in the bundle pinning region.

In the framework of the second scenario the equation for the order-disorder transition line (which is the continuation of the melting line to $t<t_{\mathrm{up}}$ ) in the bundle pinning region follows from criterion (56) as:

$$
\frac{A\left[D g_{0}(t)\right]^{3 / 2} h_{m}^{1 / 4}\left[1-h_{s v}(t)\right]^{3 / 4}}{\left[\left(1-h_{m}\right)^{3 / 2}+F_{T}(t) h_{m}^{-1 / 2}\right]^{1 / 2}}=2 \pi c_{L}^{2},
$$

i.e., the first term in Eq. (B3) disappears. If this orderdisorder line enters the single vortex pinning regions, it is described by equations that result from criterion (57). This criterion reads in explicit form

$$
A_{1} h_{s v}^{3 / 5}(t) h_{m}^{2 / 5}\left[1+\frac{F_{T}(t)}{h_{s v}^{1 / 2}\left(1-h_{m}\right)^{3 / 2}}\right]=2 \pi c_{L}^{2}
$$

for the order-disorder line in the lower single vortex pinning region, while in the upper single vortex pinning region one finds:

$$
A_{1} F_{T}(t) \frac{h_{m}^{1 / 2}}{\left(1-h_{m}\right)^{3 / 2}}+A_{1} h_{m}=2 \pi c_{L}^{2} .
$$

Here we have used that $u(a, 0)=r_{p}$ in the upper region.

In the framework of the first scenario, equations for the order-disorder line follow from condition (58) $\underline{\underline{4}}$ With the use of our approximation $f_{1}=2$, equation (24) of Ref. 4 for the order-disorder line $H_{d i s}(t)$ in the bundle pinning region can be written in the form:

$$
h_{d i s}^{1 / 2}\left(1-h_{d i s}\right)^{3 / 2}=F_{T}(t) K_{ \pm}(t),
$$

where $h_{d i s}=H_{d i s}(t) / H_{c 2}(t)$,

$$
K_{ \pm}(t)=G_{1}^{2}-1 \pm\left[\left(G_{1}^{2}-1\right)^{2}-1\right]^{1 / 2},
$$

and

$$
G_{1}=\frac{\pi^{1 / 4} G(t)\left[1-h_{s v}(t)\right]^{3 / 4}}{2^{1 / 4} c_{L}}
$$

with $G(t)$ from Eq. (44). The order-disorder line $H_{d i s}(t)$ in the single vortex pinning region is given by Eq. (22) of Ref. 4:

$$
\begin{array}{r}
h_{d i s}\left[1+\frac{F_{T}(t)}{\left(1-h_{d i s}\right)^{3 / 2}\left[h_{s v}(t)\right]^{1 / 2}}\right]^{5 / 2}= \\
2 \pi c_{L}^{2}\left(\frac{2 \pi c_{L}^{2}}{h_{s v}(t)}\right)^{3 / 2} .
\end{array}
$$

Note that apart from the factor $A_{1}$ Eq. (B11) is equivalent to Eq. (B6). 
1 D. Ertaş, D.R. Nelson, Physica C 272, 79 (1996).

2 T. Giamarchi, P. Le Doussal, Phys. Rev. B 55, 6577 (1997).

3 V. Vinokur, B. Khaykovich, E. Zeldov, M. Konczykowski, R.A. Doyle, P.H. Kes, Physica C 295, 209 (1998).

${ }^{4}$ G. P. Mikitik, E. H. Brandt, Phys. Rev. B 64, 184514 (2001).

5 Y. Radzyner, A. Shaulov, and Y. Yeshurun, Phys. Rev. B 65, 100513(R) (2002); Y. Radzyner, A. Shaulov, Y. Yeshurun, I.Felner, K. Kishio, J. Shimoyama, ibid. 65, 100503(R) (2002).

6 T. Giamarchi, P. Le Doussal, Phys. Rev. B 52, 1242 (1995).

7 T. Nattermann and S. Scheidl, Adv. Phys. 49, 607 (2000).

8 J. Kierfeld, V. Vinokur, Phys. Rev. B 61, 14928 (2000).

${ }^{9}$ G. Blatter, M.V. Feigel'man, V.B. Geshkenbein, A.I. Larkin, V.M. Vinokur, Rev. Mod. Phys. 66, 1125 (1994).

10 T.K. Worthington, M.P.A. Fisher, D.A. Huse, J. Toner, A.D. Marwick, T. Zabel, C.A. Feild, and F. Holtzberg, Phys. Rev. B 46, 11854 (1992).

11 D. Pal, S. Ramakrishnan, A.K. Grover, D. Dasgupta, B.K. Sarma, Phys. Rev. B 63, 132505 (2001).

12 N. Kobayashi, T. Nishizaki, K. Shibata, T. Sato, M. Maki, T. Sasaki, Physica C 362, 121 (2001).

13 F. Bouquet, C. Marcenat, E. Steep, R. Calemczuk, W.K. Kwok, U. Welp, G.W. Crabtree, R.A. Fisher, N.E. Phillips, A. Schilling, Nature (London) 411 , 448 (2001).

14 T. Nishizaki, K. Shibata, M. Maki, and N. Kobayashi, J. Low Temp. Phys., in print (2003).

15 K. Shibata, T. Nishizaki, T. Sasaki, N. Kobayashi, Phys. Rev. B 66, 214518 (2002).

16 M.B. Gaifullin, Y. Matsuda, N. Chikumoto, J. Shimoyama, and K. Kishio, Phys. Rev. Lett. 84, 2945 (2000).

17 C.J. van der Beek, S. Colson, M.V. Indenbom, and M. Konczykowski, Phys. Rev. Lett. 84, 4196 (2000).

18 M. Avraham, B. Khaykovich, Y. Myasoedov, M. Rappaport, H. Shtrickman, D.E. Feldman, T. Tamegai, P.H. Kes, M. Li, M. Konczykowski, Kees van der Beek, and E. Zeldov, Nature 411, 451 (2001).

19 E. H. Brandt, G. P. Mikitik, Physica C, in print (2003).

20 B. Khaykovich, M. Konczykowski, E. Zeldov, R. A. Doyle, D. Majer, P. H. Kes, and T. W. Li, Phys. Rev. B 56, R517 (1997).

21 J.A. Fendrich, W.K. Kwok, J. Giapintzakis, C.J. van der Beek, V.M. Vinokur, S. Fleshler, U. Welp, H.K. Viswanathan, G.W. Crabtree, Phys. Rev. Lett. 74, 1210 (1995).

22 L.M. Paulius, W.-K. Kwok, R.J. Olsson, A.M. Petrean, V. Tobos, J.A. Fendrich, G.W. Crabtree, C.A. Burns, S. Ferguson, Phys. Rev. B 61, R11910 (2000).

23 T. Nishizaki, T. Naito, S. Okayasu, A. Iwase, and N. Kobayashi, Phys. Rev. B 61, 3649 (2000).

24 A. I. Larkin and V. M. Vinokur, Phys. Rev. Lett. 75, 4666 (1995).

25 E.H. Brandt, Rep. Prog. Phys. 58, 1465 (1995).

26 A. Houghton, R.A. Pelcovits, and A. Sudbo, Phys. Rev. B 40, 6763 (1989).

27 E.H. Brandt, Phys. Rev. Lett. 63, 1106 (1989).

28 I.M. Babich, Yu.V. Sharlai, and G.P. Mikitik, Fiz. Nizk. Temp. 20, 227 (1994) [Low Temp. Phys. 20, 220 (1994)].

29 P.A. Lee and S.R. Shenoy, Phys. Rev. Lett. 28, 1025 (1972).

30 G.P. Mikitik, Zh. Eksp. Teor. Fiz. 101, 1042 (1992) [Sov.
Phys. JETP 74, 558 (1992)].

31 The width of the fluctuation region adjoining the line $H_{c 2}(t)$ is different for $H<G i H_{c 2}(0)$ and for $H>$ Gi $H_{c 2}(0)$, see Ref. 30 .

32 E.H. Brandt, J. Low Temp. Phys. 26, 709, 735 (1997); ibid 28, 263, 291 (1977)

33 This approximation leads only to small quantative corrections to the phase diagrams obtained in Ref. 4.

${ }^{34}$ G.P. Mikitik, Physica C 245, 287 (1995).

35 D. Li and B. Rosenstein, Phys. Rev. Lett. 86, 3618 (2001); Phys. Rev. B 65024513 (2001).

36 S. Hikami, A. Fujita, A.I. Larkin, Phys. Rev. B 44, 10400 (1991)

37 D. Li, B. Rosenstein, Phys. Rev. B 65, 220504(R) (2002).

38 M.J.W. Dodgson, V.B. Geshkenbein, H. Nordborg, G. Blatter, Phys. Rev. Lett. 80, 837 (1998).

39 M.V. Feigel'man and V.M. Vinokur, Phys. Rev. B 41, 8986 (1990).

40 L.D. Landau and E.M. Lifshitz, Theory of Elasticity, Course in Theoretical Physics Vol. 7 (Pergamon, London/Paris, 1959).

41 M.C. Marchetti, D.R. Nelson, Phys. Rev. B 41, 1910 (1990)

42 In Ref. 4, the normalization factor $\left(1-h_{s v}\right)^{3 / 2}$ [with $\left.h_{s v}=H_{s v}(t) / H_{c 2}(t)\right]$ was introduced to provide a smooth crossover of the correlation function Eq. (46) at the boundary of the single vortex pinning region $H_{s v}(T)$. In Sec. III we deal only with the bundle pinning region and so omit this factor (this has only a small effect on our results). But we shall take into account this factor in the calculations of phase diagrams in Sec. IV B, see formulas in Appendix B.

43 Note that the magnitude of the thermal fluctuations in the ideal vortex lattice, Eq. (6), practically coincides with the magnitude of these fluctuations in the lattice with pinning when the bundle pinning regime occurs. $\stackrel{4}{*}$ This is due to the fact that the main contribution to $u_{T}$ results from the modes with short wavelengths $\left(k_{\perp} \sim 1 / a\right)$, while the quenched disorder essentially distorts the lattice on the scale $R_{c}$.

44 See formulas (4.17), (4.91), (4.120) in Ref. 9, Eq. (14) in Ref. 4 , and the comment in Ref. 40.

${ }^{45}$ For $t<t_{i}$, the local minimum of $\Delta F\left(\tilde{l}_{\perp}\right)$ at $\tilde{l}_{\perp}=1$ corresponds to a transition between two liquids that differ in their density of dislocations.

46 Note that in Refs. 123 and 5, the order-disorder line was considered only in the single vortex pinning region.

47 A.I. Larkin and Yu. N. Ovchinnikov, J. Low Temp. Phys. 34, 409 (1979).

48 G.P. Mikitik, E.H. Brandt, Phys. Rev. Lett. 89, 259701 (2002).

49 See the definition of $D$, Eq. (32), and formulas (3.71) (3.74) in Ref. 9 .

50 According to the recipe given here, the function $f(h)$ in Eq. (6) for $u_{T}$ should be written in the form: $f(h)=$ $f_{1}\left[1-h_{s v}(t)\right]^{1 / 2} /(1-h)^{3 / 2}$ in the case of a vortex lattice with pinning. However, this modification leads only to small quantative corrections to the phase diagrams presented in Sec. IV B. So, similarly to Ref. 4, in the bundle pinning region we use the expression for $u_{T}$ of the ideal lattice when we write the equations below. 